\title{
ORBITAL STABILITY OF PEAKONS FOR A GENERALIZATION OF THE MODIFIED CAMASSA-HOLM EQUATION
}

\author{
XIAOCHUAN LIU, YUE LIU, PETER J. OLVER, AND CHANGZHENG QU
}

\begin{abstract}
The orbital stability of the peaked solitary-wave solutions for a generalization of the modified Camassa-Holm equation with both cubic and quadratic nonlinearities is investigated. The equation is a model of asymptotic shallow-water wave approximations to the incompressible Euler equations. It is also formally integrable in the sense of the existence of a Lax formulation and bi-Hamiltonian structure. It is demonstrated that, when the Camassa-Holm energy counteracts the effect of the modified Camassa-Holm energy, the peakon and periodic peakon solutions are orbitally stable under small perturbations in the energy space.
\end{abstract}

Key words and phrases: Camassa-Holm equation; modified Camassa-Holm equation; peakon; integrable system; orbital stability.

2000 Mathematics Subject Classification. Primary: 35B30, 35G25.

\section{INTRODUCTION}

The well-studied Camassa-Holm $(\mathrm{CH})$ equation

$$
m_{t}+2 u_{x} m+u m_{x}=0, \quad m=u-u_{x x},
$$

was originally proposed as a nonlinear model for the unidirectional propagation of the shallow water waves over a flat bottom $[1,9,18,22]$. The $\mathrm{CH}$ equation can also be derived by applying the method of tri-Hamiltonian duality to the bi-Hamiltonian representation of the Kortewegde Vries (KdV) equation, thus justifying its status as a dual integrable bi-Hamiltonian system $[17,29]$. Tri-Hamiltonian duality is based on the observation that most compatible pairs of Hamiltonian operators are, in fact, linear combinations of three mutually compatible Hamiltonian operators, and reconfiguring the operators in question will yield interesting new bi-Hamiltonian systems.

Applying tri-Hamiltonian duality to the modified Korteweg-deVries (mKdV) equation, leads to the modified Camassa-Holm $(\mathrm{mCH})$ equation with cubic nonlinearity

$$
m_{t}+\left(\left(u^{2}-u_{x}^{2}\right) m\right)_{x}=0, \quad m=u-u_{x x} .
$$

As a consequence of duality, the $\mathrm{mCH}$ equation (1.2) is formally integrable in the sense that it admits a bi-Hamiltonian structure [29] and was later shown to admit a Lax formulation [30]. Moreover, the $\mathrm{mCH}$ equation (1.2) exhibits new features, including wave breaking and blowup criteria [19] that do not appear in the original $\mathrm{CH}$ equation $(1.1)[3,5,6,7,24]$. On the other hand, since the $\mathrm{mCH}$ equation (1.2) also arises from an intrinsic (arc-length preserving) invariant planar curve flow in Euclidean geometry [19], it can be regarded as a Euclidean-invariant version of the $\mathrm{CH}$ equation (1.1), just as the mKdV equation is a Euclidean-invariant counterpart to the KdV equation from the viewpoint of curve flows in Klein geometries $[2,20]$. It is worth mentioning that besides the application of the $\mathrm{mCH}$ equation in the modeling of nonlinear water waves pointed out by Fokas [15], the present 
authors [19] showed that the scaling limit equation of (1.2), when combined with the firstorder term $\gamma u_{x}$, satisfies the short-pulse equation

$$
v_{x t}=\frac{1}{3}\left(v^{3}\right)_{x x}+\gamma v
$$

which is a model for the propagation of ultra-short light pulses in silica optical fibers [34].

More generally, applying tri-Hamiltonian duality to the bi-Hamiltonian Gardner equation

$$
u_{t}+u_{x x x}+k_{1} u^{2} u_{x}+k_{2} u u_{x}=0,
$$

the resulting dual system is the following generalized modified Camassa-Holm ( $\mathrm{gmCH}$ ) equation with both cubic and quadratic nonlinearities [16, 17]:

$$
m_{t}+k_{1}\left(\left(u^{2}-u_{x}^{2}\right) m\right)_{x}+k_{2}\left(2 u_{x} m+u m_{x}\right)=0, \quad m=u-u_{x x} .
$$

This equation, posed on the real line $x \in \mathbb{R}$ and also on the circle $x \in \mathbb{S}^{1}=\mathbb{R} / \mathbb{Z}$, i.e. subject to periodic boundary conditions, is the object of study in the present paper. It models the unidirectional propagation of the shallow-water waves over a flat bottom, where the function $u$ represents the free surface elevation, and was derived from the two-dimensional hydrodynamical equations for surface waves by Fokas [16]. Note that equation (1.5) reduces to the $\mathrm{CH}$ equation (1.1) when $k_{1}=0, k_{2}=1$, and to the $\mathrm{mCH}$ equation $(1.2)$ when $k_{1}=1$, $k_{2}=0$, respectively.

The derivation of the gmCH (1.5) via the method of tri-Hamiltonian duality reveals its status as an integrable system. Indeed, it can be written in the bi-Hamiltonian form $[28,31]$

$$
m_{t}=J \frac{\delta H_{1}}{\delta m}=K \frac{\delta H_{2}}{\delta m}
$$

where

$$
J=-k_{1} \partial_{x} m \partial_{x}^{-1} m \partial_{x}-\frac{1}{2} k_{2}\left(m \partial_{x}+\partial_{x} m\right) \quad \text { and } \quad K=-\frac{1}{4}\left(\partial_{x}-\partial_{x}^{3}\right)
$$

are compatible Hamiltonian operators, while the corresponding Hamiltonian functionals are given by

$$
H_{1}[u]=\int_{X}\left(u^{2}+u_{x}^{2}\right) d x, \quad \text { and } \quad H_{2}[u]=k_{1} I_{1}[u]+2 k_{2} I_{2}[u],
$$

where

$$
I_{1}[u]=\int_{X}\left(u^{4}+2 u^{2} u_{x}^{2}-\frac{1}{3} u_{x}^{4}\right) d x, \quad I_{2}[u]=\int_{X}\left(u^{3}+u u_{x}^{2}\right) d x .
$$

Throughout, $X=\mathbb{R}$ in the real line case, while $X=\mathbb{S}^{1}$ in the periodic case. Furthermore, a Lax pair for (1.5) was established in [31].

The gmCH equation (1.5) belongs to a novel class of physically important integrable equations. Indeed, consider the motion of a 2-dimensional, inviscid, incompressible and irrotational fluid (e.g. water) on a horizontal flat bottom located at $y=-h_{0}$, with $h_{0}$ a positive constant, and air above the free surface, whose displacement from equilibrium is represented by $\eta(t, x)$. The system of such a motion is characterized by two parameters, $\alpha=a / h_{0}$ and $\beta=h_{0}^{2} / l^{2}$, where $a$ and $l$ are typical values of the amplitude and of the wavelength of the waves. For unidirectional wave propagation, applying the physically meaningful asymptotic analysis in the shallow water regime, that is, neglecting the terms of $\mathcal{O}\left(\alpha^{4}, \alpha^{3} \beta, \beta^{2}\right)$, it is shown that the free surface $\eta(t, x)$ satisfies [16]

$$
\begin{aligned}
\eta_{t}+\eta_{x}+\alpha \eta \eta_{x}+\beta \eta_{x x x}+ & \rho_{1} \alpha^{2} \eta^{2} \eta_{x}+\alpha \beta\left(\rho_{2} \eta \eta_{x x x}+\rho_{3} \eta_{x} \eta_{x x}\right) \\
& +\rho_{4} \alpha^{3} \eta^{3} \eta_{x}+\alpha^{2} \beta\left(\rho_{5} \eta^{2} \eta_{x x x}+\rho_{6} \eta \eta_{x} \eta_{x x}+\rho_{7} \eta_{x}^{3}\right)=0
\end{aligned}
$$

where $\rho_{1}, \ldots, \rho_{7}$, are constants. Employing the following Kodama transformation

$$
\eta=u+\lambda_{1} \alpha u^{2}+\lambda_{2} \beta u_{x x}+\lambda_{3} \alpha^{2} u^{3}+\alpha \beta\left(\lambda_{4} u u_{x x}+\lambda_{5} u_{x}^{2}\right),
$$


where $\lambda_{1}, \ldots, \lambda_{5}$ are certain combinations of the $\rho_{j}$, Fokas [16] pointed out that the asymptotic model (1.9) is equivalent to the integrable gmCH equation (1.5). The appearance of an integrable equation in a class of equivalent models can reveal a great amount of information about the underlying physical system. In addition, it was argued by Fokas [15] that equation (1.5) describes the physics more accurately than its celebrated counterpart, the Gardner equation (1.4).

Furthermore, the gmCH equation (1.5) admits the following scaling-limit version

$$
v_{x t}-k_{1} v_{x}^{2} v_{x x}+k_{2}\left(v v_{x x}+\frac{1}{2} v_{x}^{2}\right)=0,
$$

which is also integrable and models the asymptotic dynamics of a short capillary-gravity wave with $v(t, x)$ denoting the fluid velocity on the surface [14]. Notably, the integrable model that, in a sense, lies midway between (1.5) and its limiting version (1.10), known as the generalized $\mu-\mathrm{CH}$ equation, was introduced in [33]:

$$
m_{t}+k_{1}\left(\left(2 \mu(u) u-u_{x}^{2}\right) m\right)_{x}+k_{2}\left(2 u_{x} m+u m_{x}\right)=0, \quad m=\mu(u)-u_{x x},
$$

where $u(t, x)$ is a real-valued spatially periodic function, with

$$
\mu(u)=\int_{\mathbb{S}^{1}} u(t, x) d x
$$

denoting its total integral.

Dual integrable nonlinear systems, such as the $\mathrm{CH}$ equation (1.1), the $\mathrm{mCH}$ equation (1.2) and the gmCH equation (1.5), exhibit nonlinear dispersion, and, in most cases, admit a remarkable variety of non-smooth soliton-like solutions, including peakons, compactons, tipons, rampons, mesaons, and so on [25]. For the $\mathrm{CH}$ equation (1.1), its single peakon has the exponential form

$$
\varphi_{c}(t, x)=a e^{-|x-c t|}
$$

with amplitude equal to the wave speed:

$$
a=c, \quad c \in \mathbb{R} .
$$

On the other hand, the periodic peakons are given by

$$
\psi_{c}(t, x)=a \cosh \left(\frac{1}{2}-(x-c t)+[x-c t]\right),
$$

where the notation $[x]$ denotes the largest integer part of the real number $x \in \mathbb{R}$; the amplitude is given by

$$
a=\frac{c}{\cosh \frac{1}{2}}, \quad c \in \mathbb{R} .
$$

The $\mathrm{mCH}$ equation (1.2) admits the single peakon of the same exponential form (1.13), but with only positive wave speeds and corresponding amplitude [19]

$$
a=\sqrt{\frac{3 c}{2}}, \quad c>0 .
$$

Similarly, its periodic peakon is given by (1.14), again with positive wave speed and amplitude [32]

$$
a=\sqrt{\frac{3 c}{1+2 \cosh ^{2} \frac{1}{2}}}, \quad c>0 .
$$

Both equations (1.1) and (1.2) also admit multi-peakon solutions of the form

$$
u(t, x)=\sum_{i=1}^{N} p_{i}(t) e^{-\left|x-q_{i}(t)\right|},
$$


although the dynamical behavior of the coefficients $p_{i}(t), q_{i}(t)$ is rather different in each instance. Indeed, for the $\mathrm{CH}$ equation they satisfy the dynamical system $[1,12,21]$

$$
\dot{p}_{i}=\sum_{j \neq i} p_{i} p_{j} \operatorname{sign}\left(q_{i}-q_{j}\right) e^{-\left|q_{i}-q_{j}\right|}, \quad \dot{q}_{i}=\sum_{j} p_{j} e^{-\left|q_{i}-q_{j}\right|}, \quad i=1, \ldots, N,
$$

whereas for the $\mathrm{mCH}$ equation [19] they satisfy

$$
\dot{p}_{i}=0, \quad \dot{q}_{i}=\frac{2}{3} p_{i}^{2}+2 \sum_{j=1}^{N} p_{j} p_{i} e^{-\left|q_{i}-q_{j}\right|}+4 \sum_{1 \leq k<i, i<j \leq N} p_{k} p_{j} e^{-\left|q_{k}-q_{j}\right|}, \quad i=1, \ldots, N .
$$

Thus, interestingly, unlike the $\mathrm{CH}$ equation, the multi-peakon amplitudes for the $\mathrm{mCH}$ equation are independent of time [19].

Recently, it was found [31] that, for $k_{1} \neq 0$, the gmCH equation (1.5) admits a single peakon of the form (1.13) with the following amplitude and restriction on the wave speed:

$$
a=\frac{3}{4} \frac{-k_{2} \pm \sqrt{k_{2}^{2}+\frac{8}{3} k_{1} c}}{k_{1}}, \quad k_{2}^{2}+\frac{8}{3} k_{1} c \geq 0 .
$$

In this paper, we shall prove that equation (1.5) also possesses periodic peakons of the form (1.14) with

$$
a=\frac{3}{2} \frac{-k_{2} \cosh \frac{1}{2} \pm \sqrt{k_{2}^{2} \cosh ^{2} \frac{1}{2}+\frac{4}{3} k_{1} c\left(1+2 \cosh ^{2} \frac{1}{2}\right)}}{k_{1}\left(1+2 \cosh ^{2} \frac{1}{2}\right)},
$$

It is worth noting that the periodic peakons of the $\mu$-integrable equation are of a manifestly different character. For example, in [33], the authors showed that the periodic peakons of the generalized $\mu$ - $\mathrm{CH}$ equation (1.11) take the following form

$$
u(t, x)=\chi_{c}(x-c t)=a \chi(x-c t),
$$

where

$$
\chi(x)=x^{2}+\frac{23}{12}, x \in\left[-\frac{1}{2}, \frac{1}{2}\right],
$$

extended periodically to the real line and

$$
a=\frac{-13 k_{2} \pm \sqrt{169 k_{2}^{2}+1200 c k_{1}}}{50 k_{1}}
$$

with $169 k_{2}^{2}+1200 c k_{1} \geq 0$.

Physically, the principal feature of the preceding peakons that their profile is smooth, except at the crest where it is continuous but the lateral tangents differ, is similar to that of the well known Stokes waves of greatest height - the traveling waves of maximum possible amplitude that are solutions to the governing equations for irrotational water waves $[4,8$, 35]. In our case, choosing different signs of the parameters $k_{1}$ and $k_{2}$ enables us to better understand how the peakons and anti-peakons interact in propagation of waves, recovering their shape and speed after a nonlinear interaction. However, if they are to be validated as physically relevant solutions, they must be dynamically stable under small perturbations. Since a small change in the height of a peakon yields another one traveling at a different speed, the appropriate notion of stability here is that of orbital stability: a wave with an initial profile close to a peakon remains close to some translate of it for all later times. That is, the shape of the wave remains approximately the same for all times.

In an innovative paper [11], using the known conservation laws of the $\mathrm{CH}$ equation and underlying features of the peakons, Constantin and Strauss proved that the single peakon solutions of the $\mathrm{CH}$ equation (1.1) are orbitally stable. Their key argument is to establish 
an inequality relating the conserved densities with the maximal values of the perturbed solutions. The Constantin-Strauss approach was recently extended to study the orbital stability of single peakons for the Degasperis-Procesi (DP) equation [26], and the $\mathrm{mCH}$ equation [32]. A variational approach for establishing the orbital stability of the $\mathrm{CH}$ peakons was introduced by Constantin and Molinet [10]. The orbital stability of trains of peakons of the $\mathrm{CH}$ equation and the $\mathrm{mCH}$ equation was explored in [13] and in [27], respectively. Stability of the periodic peakons of the $\mathrm{CH}$ equation was established by Lenells [23]. Very recently, Lenells' approach was further extended to prove the orbital stability of the periodic peakons for the $\mathrm{mCH}$ equation [32]. In [33], we were able to find an analytical method that could deal with the interaction between the different copmponents in the energy and obtained the orbital stability of periodic peakons (1.17) of the generalized $\mu$ - $\mathrm{CH}$ equation (1.11). However, for reasons explained in detail below, this analysis does not extend to the gmCH equation, which requires a new approach.

The goal of this paper is to establish the orbital stability of single peakons and periodic peakons of the $\mathrm{gmCH}$ equation (1.5). We will establish the following respective stability results.

Theorem 1.1. Assume that $k_{1}>0, k_{2}>0$ and $c \geq-\frac{3}{8} k_{2}^{2} / k_{1}$, or $k_{1}>0, k_{2} \leq 0$ and $c>\frac{2}{3} k_{2}^{2} / k_{1}$. Under either of these conditions, the peakon solutions of the gmCH equation (1.5) are orbitally stable in the energy space $H^{1}(\mathbb{R})$.

Theorem 1.2. Assume that either

$$
k_{1}>0, \quad k_{2}>0, \quad \text { and } \quad c \geq-\frac{3 k_{2}^{2} \cosh ^{2} \frac{1}{2}}{4 k_{1}\left(1+2 \cosh ^{2} \frac{1}{2}\right)},
$$

or

$$
k_{1}>0, \quad k_{2} \leq 0, \quad \text { and } \quad c>\frac{3 k_{2}^{2}}{4 k_{1}}\left(2 \cosh ^{2} \frac{1}{2}-2 \cosh \frac{1}{2}+1\right) .
$$

Then the periodic peakon solutions of the gmCH equation (1.5) are orbitally stable in the energy space $H^{1}\left(\mathbb{S}^{1}\right)$.

For the gmCH equation (1.5), the three conservation laws $H_{1}[u], H_{2}[u]$ in (1.7), along with

$$
H_{0}[u]=\int_{X} u d x
$$

will play a major role in our analysis. From the conservation law $H_{1}[u]$, it is reasonable to expect the orbital stability of (periodic) peakons for (1.5) in the sense of the energy space $H^{1}$ norm. The approach used here is motivated by the recent works $[11,23,32]$. The key issue is to establish a suitable inequality relating the maximum (and minimum) of the perturbed solution with the conserved densities, and this will rely on the introduction of a suitably constructed auxiliary function. Moreover, the corresponding equality is required to hold at the (periodic) peakons; this condition is crucial since stable (periodic) peakons must be critical points of the energy functional with the momentum constraint, and satisfy the corresponding Euler-Lagrangian equations. Since the gmCH equation (1.5) consists of two parts - the cubic $\mathrm{mCH}$ terms and the quadratic $\mathrm{CH}$ terms - this suggests choosing the required auxiliary function in the form

$$
h(t, x)=k_{1}\left(u^{2}(t, x) \mp \frac{2}{3} u(t, x) u_{x}(t, x)-\frac{1}{3} u_{x}^{2}(t, x)\right)+2 k_{2} u(t, x)
$$

for the non-periodic case $X=\mathbb{R}$, and

$$
h(t, x)=k_{1}\left(u^{2}(t, x) \pm \frac{2}{3} u_{x}(t, x) \sqrt{u^{2}(t, x)-L^{2}}-\frac{1}{3} u_{x}^{2}(t, x)-L^{2}\right)+2 k_{2} u(t, x)
$$

with $L=\min u(t, x)$, for the periodic case $X=\mathbb{S}^{1}$, respectively. 
As for the signs of $k_{1}$ and $k_{2}$, we shall consider two possibilities: (i) $k_{1}>0$ and $k_{2}>0$; (ii) $k_{1}>0$ and $k_{2} \leq 0$. In the first case, $h(t, x)$ can be estimated by the maximum (and minimum) of $u(t, x)$ using the approach in [23,32], and the stability results follow similarly. The second case is more delicate to deal with due to the interaction between two components (1.8) of the energy $H_{2}$ that have opposite signs. For peakons on the line, motivated by the idea in [33], the key observation is that the $\mathrm{mCH}$ part $I_{1}[u]$ of $H_{2}[u]$ can be dominated by the $\mathrm{CH}$ part $I_{2}[u]$ of $H_{2}[u]$, as in (1.8), in a subtle way. More precisely, the following inequality is derived:

$$
I_{1}[u] \leq \frac{4}{3} M I_{2}[u], \quad \text { where } \quad M=\max u(t, x) .
$$

While a slight different relationship was established in [33] in the periodic case for the corresponding periodic peakons, the detailed proofs are, however, rather different and neither can be deduced from the other. It is noted that the stability results in [33] rely on the construction of a concave function associated to the maximum and minimum of the perturbed solution with certain properties of periodic peakons. Our argument in the present paper, however, requires only estimating the maximum of the perturbed solutions.

In particular, in the periodic case, the generalized $\mu$-CH equation (1.11) admits the integral $\int_{\mathbb{S} 1} u_{x}^{2} d x$ as a conservation law, which is crucial in the argument to establish the orbital stability of the periodic peakons (1.17) of (1.11). But this approach does not work for the gmCH equation (1.5), due to a lack of such a conserved quantity, and thus a new approach is be required. A key observation to our case is how to estimate a uniform lower bound for the minimum of the perturbed solution $u(t, x)$. It is worth mentioning that the required lower bound depends only on the appropriate conserved densities. As a result of these insights, the orbital stability of periodic peakons in case (ii) can be established.

The outline of the paper is as follows. Section 2 is a short review on the well-posedness of the gmCH equation (1.5). In addition, the existence of periodic peakons is demonstrated rigorously in Theorem 2.1, whose proof is relegated to the Appendix. The orbital stability of the peakon solutions in the Sobolev space $H^{1}(\mathbb{R})$ is established in Section 3. In Section 4 , it is shown that the periodic peakons are dynamically stable under small perturbations in the energy space $H^{1}\left(\mathbb{S}^{1}\right)$.

Notation. Throughout the paper, the norm of a Banach space $Z$ is denoted by $\|\cdot\|_{Z}$, while $C([0, T), Z)$ denotes the class of continuous functions from the interval $[0, T)$ to $Z$. In the periodic case, we denote $\mathbb{S}^{1}=\mathbb{R} / \mathbb{Z}$ as the unit circle and regard functions on $\mathbb{S}^{1}$ as periodic on the entire line with period one. Given $T>0$, let $C_{c}^{\infty}([0, T) \times X)$ denote the space of all smooth functions with compact support on $[0, T) \times X$, which can also be viewed as the space of smooth functions on $\mathbb{R} \times X$ having compact support contained in $[0, T) \times X$. For $1 \leq p<\infty, L^{p}(X)$ denotes the space of equivalence classes of Lebesgue measurable, $p^{t h}$-power integrable, real-valued functions defined on $X$. The usual modification is in effect for $p=\infty$. The norm on $L^{p}(X)$ is written as $\|\cdot\|_{L^{p}(X)}$. For $s \geq 0$, the $L^{2}$-based Sobolev space $H^{s}$ is the subspace of those $L^{2}$ functions whose derivatives up to order $s$ all lie in $L^{2}$. The associated norm is denoted as $\|\cdot\|_{H^{s}(X)}$.

\section{Preliminaries}

In this paper, we are concerned with the Cauchy problem for the gmCH equation on both the line and the unit circle:

$$
\left\{\begin{array}{lr}
m_{t}+k_{1}\left(\left(u^{2}-u_{x}^{2}\right) m\right)_{x}+k_{2}\left(2 u_{x} m+u m_{x}\right)=0, & m(t, x)=u(t, x)-u_{x x}(t, x), \\
u(0, x)=u_{0}(x), & t>0, \quad x \in X=\mathbb{R} \text { or } \mathbb{S}^{1} .
\end{array}\right.
$$

We first formalize the notion of a strong solution. 
Definition 2.1. If $u \in C\left([0, T), H^{s}(X)\right) \cap C^{1}\left([0, T), H^{s-1}(X)\right)$, with $s>\frac{5}{2}$ and some $T>0$, satisfies (2.1), then $u$ is called a strong solution on $[0, T)$. If $u$ is a strong solution on $[0, T)$ for every $T>0$, then it is called a global strong solution.

The following local well-posedness result and properties for strong solutions on both the line and the unit circle can be established using the same approach as in [19]. The proofs are thus omitted.

Proposition 2.1. Let $u_{0} \in H^{s}(X)$ with $s>\frac{5}{2}$. Then there exists a time $T>0$ such that the initial value problem $(2.1)$ has a unique strong solution $u \in C\left([0, T), H^{s}(X)\right) \cap$ $C^{1}\left([0, T), H^{s-1}(X)\right)$. Moreover, the map $u_{0} \mapsto u$ is continuous from a neighborhood of $u_{0}$ in $H^{s}(X)$ into $C\left([0, T), H^{s}(X)\right) \cap C^{1}\left([0, T), H^{s-1}(X)\right)$.

Proposition 2.2. The Hamiltonian functionals (1.7), (1.20) are all conserved for the strong solution $u(t, x)$ obtained in Proposition 2.1, that is

$$
\frac{d}{d t} H_{0}[u]=\frac{d}{d t} H_{1}[u]=\frac{d}{d t} H_{2}[u]=0, \quad \text { for all } t \in[0, T) .
$$

Furthermore, if the initial data $m_{0}(x)=\left(1-\partial_{x}^{2}\right) u_{0}(x)$ does not change sign, then $m(t, x)$ will not change sign for any $t \in[0, T)$. It follows that if $m_{0}(x) \geq 0(\leq 0)$, then the corresponding solution $u(t, x)$ remains positive (negative) for $(t, x) \in[0, T) \times X$.

Note that the inverse operator $\left(1-\partial_{x}^{2}\right)^{-1}$ can be obtained by convolution with the corresponding Green's function, so that

$$
u=\left(1-\partial_{x}^{2}\right)^{-1} m=G * m,
$$

where

$$
G(x)=\frac{1}{2} e^{-|x|} \quad \text { for the non-periodic case } \quad X=\mathbb{R},
$$

while

$$
G(x)=\frac{\cosh \left(\frac{1}{2}-x+[x]\right)}{2 \sinh \frac{1}{2}} \quad \text { for the periodic case } \quad X=\mathbb{S}^{1},
$$

and the convolution product is defined by

$$
f * g(x)=\int_{X} f(y) g(x-y) d y .
$$

Applying the operator $\left(1-\partial_{x}^{2}\right)^{-1}$ to equation $(2.1)$, we obtain the following nonlocal nonlinear equation

$$
\begin{aligned}
u_{t}+k_{1}\left(u^{2}-\frac{1}{3} u_{x}^{2}\right) u_{x}+k_{1}\left(1-\partial_{x}^{2}\right)^{-1} & \partial_{x}\left(\frac{2}{3} u^{3}+u u_{x}^{2}\right)+\frac{1}{3} k_{1}\left(1-\partial_{x}^{2}\right)^{-1}\left(u_{x}^{3}\right) \\
& +k_{2} u u_{x}+k_{2}\left(1-\partial_{x}^{2}\right)^{-1} \partial_{x}\left(u^{2}+\frac{1}{2} u_{x}^{2}\right)=0 .
\end{aligned}
$$

The formulation (2.5) allows us to define the notion of a weak solution as follows.

Definition 2.2. Given initial data $u_{0} \in W^{1,3}(X)$, a function $u \in L_{l o c}^{\infty}\left([0, T), W_{l o c}^{1,3}(X)\right)$ is said to be a weak solution to the initial value problem (2.1) if it satisfies the following identity:

$$
\begin{aligned}
\int_{0}^{T} \int_{X} & \left\{u \partial_{t} \phi+\frac{1}{3} k_{1} u^{3} \partial_{x} \phi+\frac{1}{3} k_{1} u_{x}^{3} \phi+\frac{1}{2} k_{2} u^{2} \partial_{x} \phi+k_{1}\left[G *\left(\frac{2}{3} u^{3}+u u_{x}^{2}\right)\right] \partial_{x} \phi\right. \\
& \left.-\frac{1}{3} k_{1}\left[G * u_{x}^{3}\right] \phi+k_{2}\left[G *\left(u^{2}+\frac{1}{2} u_{x}^{2}\right)\right] \partial_{x} \phi\right\} d x d t+\int_{X} u_{0}(x) \phi(0, x) d x=0,
\end{aligned}
$$

for any smooth test function $\phi(t, x) \in C_{c}^{\infty}([0, T) \times X)$. If $u$ is a weak solution on $[0, T)$ for every $T>0$, then it is called a global weak solution.

The following theorem deals with the existence of periodic peakons for the gmCH equation (1.5) over a range of wave speeds. The proof's details can be found in the appendix. 
Theorem 2.1. For wave speeds c satisfying the inequality in (1.16), the gmCH equation (1.5) with $k_{1} \neq 0$ possesses periodic peaked traveling-wave solutions of the form (1.14). These periodic peakons are global weak solutions to (2.1) in the sense of Definition 2.2.

Remark 2.1. Since the gmCH equation (1.5) is invariant under the transformation

$$
u \longmapsto-u, \quad k_{2} \longmapsto-k_{2},
$$

it suffices to consider the peakon (1.13) and periodic peakon (1.14) with the + sign in their amplitude formulas (1.15), (1.16), an assumption that, we emphasize, will hold for the remainder of the paper.

\section{Stability of Peakons on the line}

The purpose of this section is to establish the orbital stability for the single peakon solution (1.13), (1.15) to the gmCH equation. Here, we will only consider two cases: (i) $k_{1}>0, k_{2}>0$ and (ii) $k_{1}>0, k_{2} \leq 0$. On the one hand, it is easy to check that for cases (i) with $c>0$ and (ii), the amplitude $a>0$. That is, there are only peakons, and no anti-peakons. On the other hand, for case (i) with $-\frac{3}{8} k_{2}^{2} / k_{1} \leq c<0$, the amplitude $a<0$, which implies $\varphi_{c}$ is an anti-peakon traveling from right to left.

Clearly,

$$
\max _{x \in \mathbb{R}}\left\{\left|\varphi_{c}(x)\right|\right\}=\left|\varphi_{c}(0)\right|=|a| .
$$

In addition, a direct computation leads to

$$
H_{1}\left[\varphi_{c}\right]=\left\|\varphi_{c}\right\|_{H^{1}}^{2}=2 a^{2}>0
$$

and, using (1.7), (1.8),

$$
H_{2}\left[\varphi_{c}\right]=k_{1} I_{1}\left[\varphi_{c}\right]+2 k_{2} I_{2}\left[\varphi_{c}\right]=\frac{4}{3} a^{3}\left(k_{1} a+2 k_{2}\right) .
$$

Moreover, it is worth mentioning that in case (ii), $H_{2}\left[\varphi_{c}\right]>0$ is equivalent to the condition that the wave speed $c$ satisfies

$$
c>\frac{2 k_{2}^{2}}{3 k_{1}}
$$

We are now in a position to precisely formulate Theorem 1.1. For brevity, we will concentrate our attention on case (ii).

Theorem 3.1. Let $k_{1}>0$ and $k_{2} \leq 0$. Let $\varphi_{c}$ be the peaked soliton defined in (1.13), (1.15), with wave speed satisfying (3.4). Assume that $u_{0} \in H^{s}(\mathbb{R}), s>\frac{5}{2}$, satisfies $0 \not \equiv m_{0}(x)=$ $\left(1-\partial_{x}^{2}\right) u_{0}(x) \geq 0$. Then there exists $\delta_{0}>0$, depending on $k_{1}, k_{2}, c$, and $\left\|u_{0}\right\|_{H^{s}(\mathbb{R})}$, such that if

$$
\left\|u_{0}-\varphi_{c}\right\|_{H^{1}(\mathbb{R})}<\delta, \quad 0<\delta<\delta_{0},
$$

then the corresponding positive solution $u(t, x)$ of the Cauchy problem for the gmCH equation (2.1) with initial data $u(0, x)=u_{0}(x)$ satisfies

$$
\sup _{t \in[0, T)}\left\|u(t, \cdot)-\varphi_{c}(\cdot-\xi(t))\right\|_{H^{1}(\mathbb{R})}<A \delta^{1 / 4},
$$

where $T>0$ is the maximal existence time, $\xi(t) \in \mathbb{R}$ is the point at which the solution $u(t, \cdot)$ achieves its maximum, and the constant $A>0$ depends on $k_{1}, k_{2}$, the wave speed $c$, and the norm $\left\|u_{0}\right\|_{H^{s}(\mathbb{R})}$.

Remark 3.1. As a consequence of the convolution formula (2.2), the assumptions on the initial profile $m_{0}$ imply that $u_{0}$ is strictly positive, and therefore, according to Proposition 2.2, the resulting solution $u(t, x)$ is also positive.

The proof of this theorem is based on a series of lemmas. The first two are elementary, and their proofs can be found in [11,32]. 
Lemma 3.1. For any $u \in H^{1}(\mathbb{R})$ and $\xi \in \mathbb{R}$, we have

$$
H_{1}[u]-H_{1}\left[\varphi_{c}\right]=\left\|u-\varphi_{c}(\cdot-\xi)\right\|_{H^{1}(\mathbb{R})}^{2}+4 a(u(\xi)-a) .
$$

Lemma 3.2. Let $u \in H^{s}(\mathbb{R}), s>\frac{5}{2}$. Assume $\left\|u-\varphi_{c}\right\|_{H^{1}(\mathbb{R})}<\delta$ with $0<\delta \ll 1$. Then

$$
\left|H_{1}[u]-H_{1}\left[\varphi_{c}\right]\right| \leq B \delta \quad \text { and } \quad\left|H_{2}[u]-H_{2}\left[\varphi_{c}\right]\right| \leq B \delta,
$$

where $B>0$ is a constant depending on $k_{1}, k_{2}, c$ and the norm $\|u\|_{H^{s}(\mathbb{R})}$.

Let us apply Lemmas 3.1 and 3.2 to the positive function $u(x)$ and the peakon $\varphi_{c}$ with positive amplitude $a$. Since (3.5) holds for any $\xi \in \mathbb{R}$, one can choose $\xi$ such that

$$
u(\xi)=\max _{x \in \mathbb{R}}\{u(x)\}=M,
$$

and thus

$$
\left\|u-\varphi_{c}(\cdot-\xi)\right\|_{H^{1}(\mathbb{R})}^{2}=\left(H_{1}[u]-H_{1}\left[\varphi_{c}\right]\right)-4 a(M-a) .
$$

Since the functional $H_{1}[u]$ represents the kinetic energy of the wave profile $u \in H^{1}(\mathbb{R})$, Lemma 3.1 tells that if the energy $H_{1}[u]$ and height $M$ of a wave $u \in H^{1}(\mathbb{R})$ are close to the peakon's energy and height, then the entire shape of $u$ is close to that of the peakon. Furthermore, the peakon has maximal height among all waves of fixed energy. The same remarks also apply to the $\mathrm{CH}$ equation [11] and the $\mathrm{mCH}$ equation [32]. On the other hand, in view of Lemma 3.1 and 3.2, the key task for proving the orbital stability of peakon is to control the error term which represents the difference of the maximum of the perturbed solution and the maximum of the peakon. The following Lemma is crucial to establish the estimate of such a difference.

Lemma 3.3. Suppose that $k_{1}>0, k_{2} \leq 0$ and c satisfies (3.4). For $0<u(x) \in H^{s}(\mathbb{R})$, $s>\frac{5}{2}$, let $M=\max _{x \in \mathbb{R}}\{u(x)\}$. Then there exists a $\delta_{0}>0$ depending on $k_{1}, k_{2}, c$, and $\|u\|_{H^{s}(\mathbb{R})}$, such that if $\left\|u-\varphi_{c}\right\|_{H^{1}(\mathbb{R})}<\delta$ for $0<\delta<\delta_{0}$, then

$$
0<H_{2}[u] \leq\left(\frac{4}{3} k_{1} M^{2}+2 k_{2} M\right) H_{1}[u]-\frac{4}{3} k_{1} M^{4}-\frac{4}{3} k_{2} M^{3} .
$$

Proof. First, we define the real-valued function

$$
g(x)= \begin{cases}u(x)-u_{x}(x), & x<\xi \\ u(x)+u_{x}(x), & x>\xi\end{cases}
$$

where $\xi$ satisfies (3.7). It then follows from the proof of Lemma 2 in [11] that

$$
\int_{\mathbb{R}} g^{2}(x) d x=H_{1}[u]-2 u^{2}(\xi)=H_{1}[u]-2 M^{2} .
$$

Inspired by the approach in [32], we define the function

$$
h(x)= \begin{cases}k_{1}\left(u^{2}(x)-\frac{2}{3} u(x) u_{x}(x)-\frac{1}{3} u_{x}^{2}(x)\right)+2 k_{2} u(x), & x<\xi, \\ k_{1}\left(u^{2}(x)+\frac{2}{3} u(x) u_{x}(x)-\frac{1}{3} u_{x}^{2}(x)\right)+2 k_{2} u(x), & x>\xi .\end{cases}
$$


A direct computation then shows that

$$
\begin{aligned}
\int_{\mathbb{R}} h(x) g^{2}(x) d x & \\
= & \int_{-\infty}^{\xi}\left[k_{1}\left(u^{2}-\frac{2}{3} u u_{x}-\frac{1}{3} u_{x}^{2}\right)+2 k_{2} u\right] \cdot\left(u^{2}-2 u u_{x}+u_{x}^{2}\right) d x \\
& \quad+\int_{\xi}^{\infty}\left[k_{1}\left(u^{2}+\frac{2}{3} u u_{x}-\frac{1}{3} u_{x}^{2}\right)+2 k_{2} u\right] \cdot\left(u^{2}+2 u u_{x}+u_{x}^{2}\right) d x \\
= & \int_{-\infty}^{\xi}\left[k_{1}\left(u^{4}+2 u^{2} u_{x}^{2}-\frac{1}{3} u_{x}^{4}\right)+2 k_{2}\left(u^{3}+u u_{x}^{2}\right)-\frac{8}{3} k_{1} u^{3} u_{x}-4 k_{2} u^{2} u_{x}\right] d x \\
& \quad+\int_{\xi}^{\infty}\left[k_{1}\left(u^{4}+2 u^{2} u_{x}^{2}-\frac{1}{3} u_{x}^{4}\right)+2 k_{2}\left(u^{3}+u u_{x}^{2}\right)+\frac{8}{3} k_{1} u^{3} u_{x}+4 k_{2} u^{2} u_{x}\right] d x \\
= & k_{1} \int_{\mathbb{R}}\left(u^{4}+2 u^{2} u_{x}^{2}-\frac{1}{3} u_{x}^{4}\right) d x+2 k_{2} \int_{\mathbb{R}}\left(u^{3}+u u_{x}^{2}\right) d x \\
& \quad-\frac{8}{3} k_{1} \int_{-\infty}^{\xi} u^{3} u_{x} d x+\frac{8}{3} k_{1} \int_{\xi}^{\infty} u^{3} u_{x} d x-8 k_{2} \int_{-\infty}^{\xi} u^{2} u_{x} d x+8 k_{2} \int_{\xi}^{\infty} u^{2} u_{x} d x .
\end{aligned}
$$

Thus, by (1.7), (1.8),

$$
\int_{\mathbb{R}} h(x) g^{2}(x) d x=\left(k_{1} I_{1}[u]+2 k_{2} I_{2}[u]\right)-\frac{4}{3} k_{1} M^{4}-\frac{8}{3} k_{2} M^{3}=H_{2}[u]-\frac{4}{3} k_{1} M^{4}-\frac{8}{3} k_{2} M^{3} .
$$

By our assumption, $k_{1}>0$ and $k_{2} \leq 0$, hence

$$
\begin{aligned}
h(x) & =k_{1}\left[u^{2}(x) \mp \frac{2}{3} u(x) u_{x}(x)-\frac{1}{3} u_{x}^{2}(x)\right]+2 k_{2} u(x) \\
& \leq \frac{4}{3} k_{1} u^{2}(x)+2 k_{2} u(x)=\left(\frac{4}{3} k_{1} u(x)+2 k_{2}\right) u(x) .
\end{aligned}
$$

Noting that $u(x)>0$, we argue that if $M=\max _{x \in \mathbb{R}}\{u(x)\}$ satisfies

$$
\frac{4}{3} k_{1} M+2 k_{2}>0
$$

then, for $x \in \mathbb{R}$,

$$
h(x) \leq u(x)\left(\frac{4}{3} k_{1} u(x)+2 k_{2}\right) \leq M\left(\frac{4}{3} k_{1} M+2 k_{2}\right)=\frac{4}{3} k_{1} M^{2}+2 k_{2} M,
$$

which is the crucial observation for the stability analysis of peakons. Indeed, in this case, plugging (3.9) and (3.12) into (3.10) produces

$$
\begin{aligned}
H_{2}[u]-\frac{4}{3} k_{1} M^{4}-\frac{8}{3} k_{2} M^{3}=\int_{\mathbb{R}} h(x) g^{2}(x) d x & \leq\left(\frac{4}{3} k_{1} M^{2}+2 k_{2} M\right) \int_{\mathbb{R}} g^{2}(x) d x \\
& =\left(\frac{4}{3} k_{1} M^{2}+2 k_{2} M\right) H_{1}[u]-\frac{8}{3} k_{1} M^{4}-4 k_{2} M^{3} .
\end{aligned}
$$

Therefore, (3.8) follows and the proof of the lemma will be complete.

To justify that (3.11) holds for $u$ sufficiently close to $\varphi_{c}$, we first note that, since $u(x)>0$, by the Cauchy-Schwarz inequality,

$I_{1}[u]=\int_{\mathbb{R}}\left(u^{4}+2 u^{2} u_{x}^{2}-\frac{1}{3} u_{x}^{4}\right) d x \leq \frac{4}{3} \int_{\mathbb{R}}\left(u^{4}+u^{2} u_{x}^{2}\right) d x \leq \frac{4}{3} M \int_{\mathbb{R}}\left(u^{3}+u u_{x}^{2}\right) d x=\frac{4}{3} M I_{2}[u]$.

Coupled with the fact that $I_{2}[u]>0$, we deduce that

$$
H_{2}[u] \leq\left(\frac{4}{3} k_{1} M+2 k_{2}\right) I_{2}[u] .
$$

On the other hand, in view of Lemma 3.2, if $\left\|u-\varphi_{c}\right\|_{H^{1}(\mathbb{R})}$ is small, then $H_{2}[u]$ is near $H_{2}\left[\varphi_{c}\right]$. We now conclude that if $H_{2}\left[\varphi_{c}\right]>0$, i.e. $c>\frac{2}{3} k_{2}^{2} / k_{1}$, there exists a $\delta_{0}>0$ depending on $k_{1}$, $k_{2}, c$ and $\|u\|_{H^{s}(\mathbb{R})}$ such that, for any $0<u(x) \in H^{s}(\mathbb{R}), s>\frac{5}{2}$, with $\left\|u-\varphi_{c}\right\|_{H^{1}(\mathbb{R})}<\delta$ for $0<\delta<\delta_{0}$, we have $H_{2}[u]>0$ and thus the condition (3.11) is verified. 
Lemma 3.4. Suppose that $k_{1}>0, k_{2} \leq 0$, and c satisfies (3.4). Given $0<u(x) \in H^{s}(\mathbb{R})$ with $s>\frac{5}{2}$, let $M=\max _{x \in \mathbb{R}}\{u(x)\}$. Then there exists a $\delta_{0}>0$ depending on $k_{1}, k_{2}, c$, and $\|u\|_{H^{s}(\mathbb{R})}$, such that if $\left\|u-\varphi_{c}\right\|_{H^{1}(\mathbb{R})}<\delta, 0<\delta<\delta_{0}$, then

$$
|M-a|<A \delta^{1 / 2},
$$

where $A$ is a constant depending on $k_{1}, k_{2}, c$ and $\|u\|_{H^{s}(\mathbb{R})}$.

Proof. Due to (3.8) and formula (1.7) for $H_{2}[u]=k_{1} I_{1}[u]+2 k_{2} I_{2}[u]$, we have

$$
k_{1}\left(M^{4}-H_{1}[u] M^{2}+\frac{3}{4} I_{1}[u]\right)+k_{2}\left(M^{3}-\frac{3}{2} H_{1}[u] M+\frac{3}{2} I_{2}[u]\right) \leq 0 .
$$

Define the quartic polynomial

$$
P(z)=k_{1}\left(z^{4}-H_{1}[u] z^{2}+\frac{3}{4} I_{1}[u]\right)+k_{2}\left(z^{3}-\frac{3}{2} H_{1}[u] z+\frac{3}{2} I_{2}[u]\right) .
$$

Noticing that

$$
H_{1}\left[\varphi_{c}\right]=2 a^{2}, \quad I_{1}\left[\varphi_{c}\right]=\frac{4}{3} a^{4}, \quad I_{2}\left[\varphi_{c}\right]=\frac{4}{3} a^{3},
$$

one can define another quartic polynomial

$$
\begin{aligned}
P_{0}(z) & =k_{1}\left(z^{4}-H_{1}\left[\varphi_{c}\right] z^{2}+\frac{3}{4} I_{1}\left[\varphi_{c}\right]\right)+k_{2}\left(z^{3}-\frac{3}{2} H_{1}\left[\varphi_{c}\right] z+\frac{3}{2} I_{2}\left[\varphi_{c}\right]\right) \\
& =\left[k_{1} z^{2}+\left(2 k_{1} a+k_{2}\right) z+a\left(k_{1} a+2 k_{2}\right)\right](z-a)^{2} .
\end{aligned}
$$

A direct calculation using (3.14) and (3.15) shows that

$$
P_{0}(M)=P(M)+k_{1} M^{2}\left(H_{1}[u]-H_{1}\left[\varphi_{c}\right]\right)+\frac{3}{2} k_{2} M\left(H_{1}[u]-H_{1}\left[\varphi_{c}\right]\right)-\frac{3}{4}\left(H_{2}[u]-H_{2}\left[\varphi_{c}\right]\right),
$$

which together with (3.4), (3.11), (3.13), and (3.15) implies

$$
\begin{aligned}
a\left(k_{1} a+2 k_{2}\right)(M-a)^{2} & \leq\left(k_{1} M^{2}+\frac{3}{2} k_{2} M\right)\left(H_{1}[u]-H_{1}\left[\varphi_{c}\right]\right)-\frac{3}{4}\left(H_{2}[u]-H_{2}\left[\varphi_{c}\right]\right) \\
& \leq k_{1} M^{2}\left|H_{1}[u]-H_{1}\left[\varphi_{c}\right]\right|+\frac{3}{4}\left|H_{2}[u]-H_{2}\left[\varphi_{c}\right]\right| .
\end{aligned}
$$

On the other hand, since

$$
H_{1}[u]-2 M^{2}=\int_{\mathbb{R}} g^{2}(x) d x \geq 0, \quad \text { we have } \quad 0<M^{2} \leq \frac{1}{2} H_{1}[u] \leq A,
$$

where $A$ depends on $k_{1}, k_{2}, c$ and $\|u\|_{H^{s}(\mathbb{R})}$. Hence, in view of (3.16), (3.17), and Lemma 3.2 , we conclude that

$$
|M-a| \leq A \sqrt{A\left|H_{1}[u]-H_{1}\left[\varphi_{c}\right]\right|+\left|H_{2}[u]-H_{2}\left[\varphi_{c}\right]\right|} \leq A \delta^{1 / 2} .
$$

This completes the proof of Lemma 3.4.

Proof of Theorem 3.1. Given initial data $u_{0}(x) \in H^{s}(\mathbb{R})$ satisfying the hypotheses, let $u \in C\left([0, T), H^{s}(\mathbb{R})\right) \cap C^{1}\left([0, T), H^{s-1}(\mathbb{R})\right)$ be the corresponding positive solution of the Cauchy problem (2.1) on the line, with maximal existence time $T>0$. Since $H_{1}$ and $H_{2}$ are both conserved, that means

$$
H_{1}[u(t, \cdot)]=H_{1}\left[u_{0}\right] \quad \text { and } \quad H_{2}[u(t, \cdot)]=H_{2}\left[u_{0}\right], \quad t \in[0, T) .
$$

Since the assumptions of Lemma 3.4 are satisfied for $u(t, \cdot), t \in[0, T)$, with a positive constant $A$ depending on $k_{1}, k_{2}, c$ and $\left\|u_{0}\right\|_{H^{s}(\mathbb{R})}$, we have

$$
|u(t, \xi(t))-a|<A \delta^{1 / 2}, \quad t \in[0, T),
$$

where $u(t, \xi(t))=M(t)=\max _{x \in \mathbb{R}}\{u(t, x)\}$. Due to (3.18) and Lemma 3.1,

$$
\left\|u(t, \cdot)-\varphi_{c}(\cdot-\xi(t))\right\|_{H^{1}(\mathbb{R})}^{2}=H_{1}\left[u_{0}\right]-H_{1}\left[\varphi_{c}\right]-4 a(u(t, \xi(t))-a), \quad t \in[0, T) .
$$

Combining the above estimates, we conclude that

$$
\| u(t, \cdot)-\varphi_{c}\left(\cdot-\xi(t) \|_{H^{1}(\mathbb{R})} \leq \sqrt{\left|H_{1}\left[u_{0}\right]-H_{1}\left[\varphi_{c}\right]\right|+4 a|u(t, \xi(t))-a|}<A \delta^{1 / 4}, \quad t \in[0, T),\right.
$$

which thus completes the proof of Theorem 3.1. 
Remark 3.2. In the proof, it follows from the estimate (3.16) that the peakons are energy minimizers with a fixed invariant $H_{2}$, which reveals their stability. In fact, if $H_{2}[u]=H_{2}\left[\varphi_{c}\right]$, we deduce from (3.16) that $H_{1}[u] \geq H_{1}\left[\varphi_{c}\right]$. The same remark applies to the CH, DP, and $\mathrm{mCH}$ equations and demonstrates that their peakons are also energy minimizers with corresponding fixed invariants [11, 26, 27, 32].

Next, for case (i) with positive wave speed $c$, we have the corresponding stability result.

Theorem 3.2. Assume that $k_{1}>0$ and $k_{2}>0$. Let $\varphi_{c}$ be the peaked soliton defined in (1.13), (1.15), traveling with speed $c>0$. Then $\varphi_{c}$ is orbitally stable in the following sense. Given initial data $u_{0} \in H^{s}(\mathbb{R}), s>\frac{5}{2}$, with $0 \not \equiv m_{0}(x)=\left(1-\partial_{x}^{2}\right) u_{0}(x) \geq 0$, there exists $\delta_{0}>0$, depending on $k_{1}, k_{2}, c$, and $\left\|u_{0}\right\|_{H^{s}(\mathbb{R})}$, such that if

$$
\left\|u_{0}-\varphi_{c}\right\|_{H^{1}(\mathbb{R})}<\delta, \quad 0<\delta<\delta_{0},
$$

then the corresponding solution $u(t, x)$ of the Cauchy problem for the gmCH equation (2.1) satisfies

$$
\sup _{t \in[0, T)}\left\|u(t, \cdot)-\varphi_{c}(\cdot-\xi(t))\right\|_{H^{1}(\mathbb{R})}<A \delta^{1 / 4}
$$

where $T>0$ is the maximal existence time, $\xi(t) \in \mathbb{R}$ is the maximum point of the function $u(t, \cdot)$, and the constant $A>0$ depends on $k_{1}, k_{2}$, the wave speed $c$, and the norm $\left\|u_{0}\right\|_{H^{s}(\mathbb{R})}$.

We first claim that, since $k_{1}>0$ and $k_{2}>0$, for the positive solution $u(t, x)$, the following key inequality holds:

$h(x)=k_{1}\left(u(x)^{2} \mp \frac{2}{3} u(x) u_{x}(x)-\frac{1}{3} u_{x}^{2}(x)\right)+2 k_{2} u(x) \leq \frac{4}{3} k_{1} u^{2}(x)+2 k_{2} u(x) \leq \frac{4}{3} k_{1} M^{2}+2 k_{2} M$.

With this in hand, the remainder of the proof is similar to that in our previous paper [32], and we omit it here for the sake of brevity.

Finally, invariance of the gmCH equation under the transformation $u \rightarrow-u$ immediately implies the corresponding stability result for anti-peakons.

Theorem 3.3. Let $k_{1}>0$ and $k_{2}>0$. Let $\varphi_{c}$ be the peaked soliton defined in (1.13), (1.15), traveling with speed $-\frac{3}{8} k_{2}^{2} / k_{1} \leq c<0$. Then $\varphi_{c}$ is orbitally stable in the following sense. Given initial data $u_{0} \in H^{s}(\mathbb{R}), s>\frac{5}{2}$, satisfying $0 \not \equiv m_{0}(x)=\left(1-\partial_{x}^{2}\right) u_{0}(x) \leq 0$, there exists $\delta_{0}>0$, depending on $k_{1}, k_{2}, c$, and $\left\|u_{0}\right\|_{H^{s}(\mathbb{R})}$, such that if

$$
\left\|u_{0}-\varphi_{c}\right\|_{H^{1}(\mathbb{R})}<\delta, \quad 0<\delta<\delta_{0},
$$

then the corresponding solution $u(t, x)$ of the Cauchy problem for the gmCH equation (2.1) satisfies

$$
\sup _{t \in[0, T)}\left\|u(t, \cdot)-\varphi_{c}(\cdot-\xi(t))\right\|_{H^{1}(\mathbb{R})}<A \delta^{1 / 4},
$$

where $T>0$ is the maximal existence time, $\xi(t) \in \mathbb{R}$ is the minimum point of the solution $u(t, \cdot)$, and the constant $A>0$ depends on $k_{1}, k_{2}$, the wave speed $c$, and the norm $\left\|u_{0}\right\|_{H^{s}(\mathbb{R})}$.

\section{Stability of Periodic Peakons}

In this section, we study the orbital stability of the periodic peakons (1.14), (1.16) of the gmCH equation (2.1). We will identify functions on $\mathbb{S}^{1}=\mathbb{R} / \mathbb{Z}$ as periodic functions on the whole line of period one. Hence, we write the periodic peakon in the form

$$
\psi_{c}(t, x)=a \psi(x-c t),
$$

where, in view of Remark 2.1, $a$ is defined by (1.16) with the + sign, while $\psi(x)$ represents the 1-periodic function such that

$$
\psi(x)=\cosh \left(\frac{1}{2}-x\right) \quad \text { for } \quad 0 \leq x<1 .
$$


As before, we will consider two cases: (i) $k_{1}>0, k_{2}>0$, and (ii) $k_{1}>0, k_{2} \leq 0$. Note that the case (i) contains periodic peakons with both positive amplitude and negative amplitude, whereas in case (ii), they have only positive amplitude.

According to $(4.2), \psi(x)$ is continuous on $\mathbb{S}^{1}$ with peak at $x=0$, and hence, for the periodic peakon with positive amplitude $a$, its maximum and minimum values are, respectively,

$$
M_{\psi_{c}}=\max _{x \in \mathbb{S}^{1}}\left\{\psi_{c}(x)\right\}=a \psi(0)=a \cosh \frac{1}{2}, \quad L_{\psi_{c}}=\min _{x \in \mathbb{S}^{1}}\left\{\psi_{c}(x)\right\}=a \psi\left(\frac{1}{2}\right)=a .
$$

On the other hand, $\psi(x)$ is smooth on $(0,1)$, and satisfies

$$
\psi^{\prime}(x)=-\sinh \left(\frac{1}{2}-x\right), \quad \psi^{\prime \prime}(x)=\psi(x)-2 \sinh \frac{1}{2} \delta(x),
$$

where $\delta$ denotes the Dirac distribution. Thus, by direct evaluation, the values of the conservation laws for the periodic peakon (4.1) are given by

$$
\begin{aligned}
& H_{0}\left[\psi_{c}\right]=a \int_{\mathbb{S}^{1}} \cosh \left(\frac{1}{2}-x\right) d x=2 a \sinh \frac{1}{2}, \\
& H_{1}\left[\psi_{c}\right]=a^{2} \int_{\mathbb{S}^{1}}\left(\psi^{2}+\psi_{x}^{2}\right) d x=a^{2} \int_{\mathbb{S}^{1}}\left(\psi^{2}-\psi_{x x} \psi\right) d x=2 a^{2} \sinh \frac{1}{2} \cosh \frac{1}{2} .
\end{aligned}
$$

Furthermore,

$$
\begin{aligned}
I_{1}\left[\psi_{c}\right] & =a^{4} \int_{\mathbb{S}^{1}}\left(\psi^{4}+2 \psi^{2} \psi_{x}^{2}-\frac{1}{3} \psi_{x}^{4}\right) d x=a^{4} \int_{-\frac{1}{2}}^{\frac{1}{2}}\left(\cosh ^{4} x+2 \cosh ^{2} x \sinh ^{2} x-\frac{1}{3} \sinh ^{4} x\right) d x \\
& =a^{4} \int_{-\frac{1}{2}}^{\frac{1}{2}}\left(\frac{1}{3} \cosh (4 x)+\frac{2}{3} \cosh (2 x)\right) d x=\frac{2}{3} a^{4} \sinh \frac{1}{2}\left(2 \cosh ^{3} \frac{1}{2}+\cosh \frac{1}{2}\right), \\
I_{2}\left[\psi_{c}\right] & =a^{3} \int_{\mathbb{S}^{1}}\left(\psi^{3}+\psi \psi_{x}^{2}\right) d x=a^{3} \int_{-\frac{1}{2}}^{\frac{1}{2}}\left(\cosh (3 x)-2 \cosh x \sinh ^{2} x\right) d x \\
& =2 a^{3}\left(\frac{2}{3} \sinh ^{3} \frac{1}{2}+\sinh \frac{1}{2}\right),
\end{aligned}
$$

and hence

$$
\begin{aligned}
H_{2}\left[\psi_{c}\right] & =k_{1} I_{1}\left[\psi_{c}\right]+2 k_{2} I_{2}\left[\psi_{c}\right] \\
& =\frac{2}{3} k_{1} a^{4} \sinh \frac{1}{2}\left(2 \cosh ^{3} \frac{1}{2}+\cosh \frac{1}{2}\right)+4 k_{2} a^{3}\left(\frac{2}{3} \sinh ^{3} \frac{1}{2}+\sinh \frac{1}{2}\right) .
\end{aligned}
$$

The following result is a reformulated version of the second case (1.19) of Theorem 1.2.

Theorem 4.1. Assume that $k_{1}>0$ and $k_{2} \leq 0$. Let $\psi_{c}$ be the periodic peaked soliton defined in (4.1) with the traveling wave speed c satisfying the inequality in (1.19). Then $\psi_{c}$ is orbitally stable in the following sense. Suppose that $u_{0} \in H^{s}\left(\mathbb{S}^{1}\right), s>\frac{5}{2}$, with $0 \not \equiv m_{0}=$ $\left(1-\partial_{x}^{2}\right) u_{0}(x) \geq 0$. Let $T>0$ be the maximal existence time of the corresponding periodic solution $u(t, x) \in C\left([0, T), H^{s}\left(\mathbb{S}^{1}\right)\right)$ with initial data $u_{0}$. Then, for every $\epsilon>0$, there is a $\delta>0$ such that if

$$
\left\|u_{0}-\psi_{c}\right\|_{H^{1}\left(\mathbb{S}^{1}\right)}<\delta
$$

then

$$
\left\|u(t, \cdot)-\psi_{c}(\cdot-\xi(t))\right\|_{H^{1}\left(\mathbb{S}^{1}\right)}<\epsilon \quad \text { for } \quad t \in[0, T),
$$

where $\xi(t)$ is any point where the function $u(t, \cdot)$ attains its maximum.

Remark 4.1. Due to the convolution formula (2.2) with the Green's function (2.4), the assumption on $m_{0}$ implies that $u_{0}$ is strictly positive, and hence, by Proposition 2.2, the corresponding solution $u(t, x)$ is also strictly positive.

The proof of Theorem 4.1 will be carried out through a series of lemmas. We first consider the expansion of the conservation law $H_{1}$ around the peakon $\psi_{c}$ in the $H^{1}\left(\mathbb{S}^{1}\right)$-norm. 
Lemma 4.1. For any $u \in H^{1}\left(\mathbb{S}^{1}\right)$ and $\xi \in \mathbb{R}$, we have

$$
H_{1}[u]-H_{1}\left[\psi_{c}\right]=\left\|u-\psi_{c}(\cdot-\xi)\right\|_{H^{1}\left(\mathbb{S}^{1}\right)}^{2}+4 a \sinh \left(\frac{1}{2}\right)\left(u(\xi)-M_{\psi_{c}}\right) .
$$

Proof. Using the second formulas in (4.4) and (4.5), we calculate

$$
\begin{aligned}
\| u-\psi_{c}(\cdot & -\xi) \|_{H^{1}\left(\mathbb{S}^{1}\right)}^{2}=\int_{\mathbb{S}^{1}}\left(u(x)-\psi_{c}(x-\xi)\right)^{2} d x+\int_{\mathbb{S}^{1}}\left(u_{x}(x)-\partial_{x} \psi_{c}(x-\xi)\right)^{2} d x \\
& =H_{1}[u]+H_{1}\left[\psi_{c}\right]-2 a \int_{\mathbb{S}^{1}} u(x+\xi) \psi(x) d x+2 a \int_{\mathbb{S}^{1}} u(x+\xi) \psi_{x x}(x) d x \\
& =H_{1}[u]+H_{1}\left[\psi_{c}\right]-4 a \sinh \left(\frac{1}{2}\right) u(\xi) \\
& =H_{1}[u]-H_{1}\left[\psi_{c}\right]-4 a \sinh \left(\frac{1}{2}\right)\left(u(\xi)-a \cosh \frac{1}{2}\right) \\
& =H_{1}[u]-H_{1}\left[\psi_{c}\right]-4 a \sinh \left(\frac{1}{2}\right)\left(u(\xi)-M_{\psi_{c}}\right),
\end{aligned}
$$

which establishes (4.7).

Next, we state, without proof, a periodic version of Lemma 3.2, establishing the continuity of the three conservation laws (1.7), (1.20), in the $H^{1}$-norm.

Lemma 4.2. Let $u \in H^{s}\left(\mathbb{S}^{1}\right), s>\frac{5}{2}$. Assume $\left\|u-\psi_{c}\right\|_{H^{1}\left(\mathbb{S}^{1}\right)}<\delta$ with $0<\delta \ll 1$. Then

$$
\left|H_{i}[u]-H_{i}\left[\psi_{c}\right]\right| \leq C \delta, \quad i=0,1,2,
$$

where $C>0$ is a constant depending on $k_{1}, k_{2}, c>0$ and $\|u\|_{H^{s}\left(\mathbb{S}^{1}\right)}$.

To proceed, it is observed from (4.4) that the periodic peakon $\psi_{c}$ satisfies the following differential equation

$$
\partial_{x} \psi_{c}(x)= \begin{cases}-\sqrt{\psi_{c}^{2}-L_{\psi_{c}}^{2}}, & 0<x \leq \frac{1}{2}, \\ \sqrt{\psi_{c}^{2}-L_{\psi_{c}}^{2}}, & \frac{1}{2} \leq x<1 .\end{cases}
$$

Let $0<u(x) \in H^{s}\left(\mathbb{S}^{1}\right) \subset C^{2}\left(\mathbb{S}^{1}\right), s>\frac{5}{2}$, and write

$$
M=M_{u}=\max _{x \in \mathbb{S}^{1}}\{u(x)\}=u(\xi), \quad L=L_{u}=\min _{x \in \mathbb{S}^{1}}\{u(x)\}=u(\eta),
$$

for some $\xi, \eta \in \mathbb{S}^{1}$. We now define the real function

$$
g(x)= \begin{cases}u_{x}+\sqrt{u^{2}-L^{2}}, & \xi<x \leq \eta \\ u_{x}-\sqrt{u^{2}-L^{2}}, & \eta \leq x<\xi+1,\end{cases}
$$

which is extended periodically to the entire line. A direct computation [23] yields

$$
\int_{\mathbb{S}^{1}} g^{2}(x) d x=2 L^{2} \log \left(\frac{M+\sqrt{M^{2}-L^{2}}}{L}\right)-2 M \sqrt{M^{2}-L^{2}}-L^{2}+H_{1}[u] .
$$

In addition, motivated by [32], we define the another auxiliary real function

$$
h(x)= \begin{cases}k_{1}\left(u^{2}+\frac{2}{3} u_{x} \sqrt{u^{2}-L^{2}}-\frac{1}{3} u_{x}^{2}-L^{2}\right)+2 k_{2} u, & \xi<x \leq \eta, \\ k_{1}\left(u^{2}-\frac{2}{3} u_{x} \sqrt{u^{2}-L^{2}}-\frac{1}{3} u_{x}^{2}-L^{2}\right)+2 k_{2} u, & \eta \leq x<\xi+1\end{cases}
$$


and extend it periodically to the entire line. Then

$$
\begin{array}{rl}
\int_{\mathbb{S} 1} & h(x) g^{2}(x) d x \\
= & \int_{\xi}^{\eta}\left(k_{1}\left(u^{2}+\frac{2}{3} u_{x} \sqrt{u^{2}-L^{2}}-\frac{1}{3} u_{x}^{2}-L^{2}\right)+2 k_{2} u\right)\left(u_{x}+\sqrt{u^{2}-L^{2}}\right)^{2} d x \\
& \quad+\int_{\eta}^{\xi+1}\left(k_{1}\left(u^{2}-\frac{2}{3} u_{x} \sqrt{u^{2}-L^{2}}-\frac{1}{3} u_{x}^{2}-L^{2}\right)+2 k_{2} u\right)\left(u_{x}-\sqrt{u^{2}-L^{2}}\right)^{2} d x \\
= & J_{1}+J_{2} .
\end{array}
$$

A straightforward computation leads to

$$
\begin{aligned}
J_{1}=\int_{\xi}^{\eta} & k_{1}\left(u^{2}+\frac{2}{3} u_{x} \sqrt{u^{2}-L^{2}}-\frac{1}{3} u_{x}^{2}-L^{2}\right)\left(u_{x}^{2}+2 u_{x} \sqrt{u^{2}-L^{2}}+u^{2}-L^{2}\right) d x \\
& +\int_{\xi}^{\eta} 2 k_{2} u\left(u_{x}^{2}+2 u_{x} \sqrt{u^{2}-L^{2}}+u^{2}-L^{2}\right) d x \\
= & k_{1} \int_{\xi}^{\eta}\left(u^{4}+2 u^{2} u_{x}^{2}-\frac{1}{3} u_{x}^{4}\right) d x-k_{1} L^{2} \int_{\xi}^{\eta}\left(u^{2}+u_{x}^{2}\right) d x-k_{1} L^{2} \int_{\xi}^{\eta} g^{2}(x) d x \\
& +\frac{8}{3} k_{1} \int_{\xi}^{\eta} u^{2} u_{x} \sqrt{u^{2}-L^{2}} d x-\frac{2}{3} k_{1} L^{2} \int_{\xi}^{\eta} u_{x} \sqrt{u^{2}-L^{2}} d x \\
& +2 k_{2} \int_{\xi}^{\eta}\left(u^{3}+u u_{x}^{2}\right) d x+4 k_{2} \int_{\xi}^{\eta} u u_{x} \sqrt{u^{2}-L^{2}} d x-2 k_{2} L^{2} \int_{\xi}^{\eta} u d x .
\end{aligned}
$$

Using the identities

$$
\begin{aligned}
u^{2} u_{x} \sqrt{u^{2}-L^{2}} & =\frac{1}{4} \frac{d}{d x}\left(u\left(u^{2}-L^{2}\right)^{3 / 2}\right)+\frac{1}{4} L^{2} u_{x} \sqrt{u^{2}-L^{2}} \\
u u_{x} \sqrt{u^{2}-L^{2}} & =\frac{1}{3} \frac{d}{d x}\left(u^{2}-L^{2}\right)^{3 / 2}
\end{aligned}
$$

we find

$$
\begin{aligned}
J_{1}= & k_{1} \int_{\xi}^{\eta}\left(u^{4}+2 u^{2} u_{x}^{2}-\frac{1}{3} u_{x}^{4}\right) d x-k_{1} L^{2} \int_{\xi}^{\eta}\left(u^{2}+u_{x}^{2}\right) d x-k_{1} L^{2} \int_{\xi}^{\eta} g^{2}(x) d x \\
& -\frac{2}{3} k_{1} M\left(M^{2}-L^{2}\right)^{3 / 2}+2 k_{2} \int_{\xi}^{\eta}\left(u^{3}+u u_{x}^{2}\right) d x-\frac{4}{3} k_{2}\left(M^{2}-L^{2}\right)^{3 / 2}-2 k_{2} L^{2} \int_{\xi}^{\eta} u d x .
\end{aligned}
$$

In a similar manner, we have

$$
\begin{aligned}
& J_{2}=k_{1} \int_{\eta}^{\xi+1}\left(u^{4}+2 u^{2} u_{x}^{2}-\frac{1}{3} u_{x}^{4}\right) d x-k_{1} L^{2} \int_{\eta}^{\xi+1}\left(u^{2}+u_{x}^{2}\right) d x-k_{1} L^{2} \int_{\eta}^{\xi+1} g^{2}(x) d x \\
& -\frac{2}{3} k_{1} M\left(M^{2}-L^{2}\right)^{3 / 2}+2 k_{2} \int_{\eta}^{\xi+1}\left(u^{3}+u u_{x}^{2}\right) d x-\frac{4}{3} k_{2}\left(M^{2}-L^{2}\right)^{3 / 2}-2 k_{2} L^{2} \int_{\eta}^{\xi+1} u d x .
\end{aligned}
$$

Recalling the definitions (1.7) of $H_{1}[u], H_{2}[u]$,

$$
\begin{gathered}
\int_{\mathbb{S}^{1}} h(x) g^{2}(x) d x=k_{1} I_{1}[u]- \\
k_{1} L^{2} H_{1}[u]-k_{1} L^{2} \int_{\mathbb{S}^{1}} g^{2}(x) d x-\frac{4}{3} k_{1} M\left(M^{2}-L^{2}\right)^{3 / 2} \\
+2 k_{2} I_{2}[u]-\frac{8}{3} k_{2}\left(M^{2}-L^{2}\right)^{3 / 2}-2 k_{2} L^{2} \int_{\mathbb{S}^{1}} u d x \\
=-k_{1} L^{2} \int_{\mathbb{S}^{1}} g^{2}(x) d x-\frac{4}{3} k_{1} M\left(M^{2}-L^{2}\right)^{3 / 2}-\frac{8}{3} k_{2}\left(M^{2}-L^{2}\right)^{3 / 2} \\
+H_{2}[u]-k_{1} L^{2} H_{1}[u]-2 k_{2} L^{2} H_{0}[u] .
\end{gathered}
$$


The assumption $k_{1}>0$ and $k_{2} \leq 0$ implies

$$
\begin{aligned}
h(x) & =k_{1}\left(u^{2}(x) \pm \frac{2}{3} u_{x}(x) \sqrt{u^{2}(x)-L^{2}}-\frac{1}{3} u_{x}^{2}(x)-L^{2}\right)+2 k_{2} u(x) \\
& \leq k_{1}\left(\frac{4}{3} u^{2}(x)-\frac{4}{3} L^{2}\right)+2 k_{2} u(x)=u(x)\left(\frac{4}{3} k_{1} u(x)+2 k_{2}\right)-\frac{4}{3} k_{1} L^{2} .
\end{aligned}
$$

This, if the positive function $u(x)$ with minimum $L$ satisfies

$$
\frac{4}{3} k_{1} L+2 k_{2}>0
$$

then

$$
h(x) \leq M\left(\frac{4}{3} k_{1} M+2 k_{2}\right)-\frac{4}{3} k_{1} L^{2}=\frac{4}{3} k_{1}\left(M^{2}-L^{2}\right)+2 k_{2} M .
$$

Since $u(x)>0$, the convolution formula (2.2), (2.4) implies that

$$
L=\min _{x \in \mathbb{S}^{1}}\{u(x)\} \geq \frac{1}{2 \sinh \frac{1}{2}} \int_{\mathbb{S}^{1}} m(z) d z=\frac{H_{0}[u]}{2 \sinh \frac{1}{2}} .
$$

Note that the equation holds at $u(x)=\psi_{c}(x)$. On the other hand, thanks to Lemma 4.2, we argue that if

$$
\frac{2}{3 \sinh \frac{1}{2}} k_{1} H_{0}\left[\psi_{c}\right]+2 k_{2}>0
$$

then there is a $\delta>0$ small enough, such that for $u(x)$ satisfying $\left\|u-\psi_{c}\right\|_{H^{1}\left(\mathbb{S}^{1}\right)}<\delta$, one has

$$
\frac{2}{3 \sinh \frac{1}{2}} k_{1} H_{0}[u]+2 k_{2}>0,
$$

which, along with (4.15), leads to (4.13) and

$$
\frac{4}{3} k_{1} u(x)+2 k_{2}>0, \quad x \in \mathbb{S}^{1},
$$

and this establishes the inequality (4.14). In view of (4.5), the inequality (4.16) is equivalent to the following inequality about the amplitude $a$ :

$$
\frac{4}{3} k_{1} a+2 k_{2}>0
$$

which implies that the wave speed $c$ satisfies

$$
c>\frac{3 k_{2}^{2}}{4 k_{1}}\left(2 \cosh ^{2} \frac{1}{2}-2 \cosh \frac{1}{2}+1\right) .
$$

In view of (4.12) and (4.14), we obtain

$$
\begin{array}{r}
-k_{1} L^{2} \int_{\mathbb{S}^{1}} g^{2}(x) d x-\frac{4}{3} k_{1} M\left(M^{2}-L^{2}\right)^{3 / 2}-\frac{8}{3} k_{2}\left(M^{2}-L^{2}\right)^{3 / 2}+H_{2}[u]-k_{1} L^{2} H_{1}[u] \\
-2 k_{2} L^{2} H_{0}[u] \leq \frac{4}{3} k_{1}\left(M^{2}-L^{2}\right) \int_{\mathbb{S}^{1}} g^{2}(x) d x+2 k_{2} M \int_{\mathbb{S}^{1}} g^{2}(x) d x,
\end{array}
$$

which, combined with (4.10), yields the following inequality:

$$
\begin{aligned}
0 \leq F_{u}(M, L)= & \left(k_{1}\left(\frac{4}{3} M^{2}-\frac{1}{3} L^{2}\right)+2 k_{2} M\right)\left(2 L^{2} \log \frac{M+\sqrt{M^{2}-L^{2}}}{L}\right) \\
& -2 M \sqrt{M^{2}-L^{2}}-L^{2}+\frac{4}{3} k_{1} M\left(M^{2}-L^{2}\right)^{3 / 2}+\frac{8}{3} k_{2}\left(M^{2}-L^{2}\right)^{3 / 2} \\
& -H_{2}[u]+\left(k_{1}\left(\frac{4}{3} M^{2}+\frac{2}{3} L^{2}\right)+2 k_{2} M\right) H_{1}[u]+2 k_{2} L^{2} H_{0}[u] .
\end{aligned}
$$

Note that the function $F_{u}$ depends on $u$ only through the values of the three conservation laws $H_{0}[u], H_{1}[u], H_{2}[u]$. This establishes the following lemma. 
Lemma 4.3. Assume that $k_{1}>0$ and $k_{2} \leq 0$. Then, for wave speed $c$ satisfying (1.19), there exists $\delta>0$ such that if $\left\|u-\psi_{c}\right\|_{H^{1}\left(\mathbb{S}^{1}\right)}<\delta$, then

$$
k_{1} H_{0}[u]+3 k_{2} \sinh \frac{1}{2}>0 \quad \text { and } \quad F_{u}\left(M_{u}, L_{u}\right) \geq 0,
$$

where $M_{u}=\max _{x \in \mathbb{S}^{1}}\{u(x)\}, L_{u}=\min _{x \in \mathbb{S}^{1}}\{u(x)\}$.

The next lemma summarizes some properties of the function (4.17) when $u$ is the periodic peakon $\psi_{c}$.

Lemma 4.4. Assume that $k_{1}>0, k_{2} \leq 0$ and the wave speed $c$ satisfies (1.19). Then

$$
\begin{aligned}
F_{\psi_{c}}\left(M_{\psi_{c}}, L_{\psi_{c}}\right) & =0, \quad \frac{\partial F_{\psi_{c}}}{\partial M}\left(M_{\psi_{c}}, L_{\psi_{c}}\right)=0, \quad \frac{\partial F_{\psi_{c}}}{\partial L}\left(M_{\psi_{c}}, L_{\psi_{c}}\right)=0, \\
\frac{\partial^{2} F_{\psi_{c}}}{\partial M^{2}}\left(M_{\psi_{c}}, L_{\psi_{c}}\right) & =-\frac{32}{3} k_{1} a^{2} \sinh \frac{1}{2} \cosh \frac{1}{2}-8 k_{2} a \sinh \frac{1}{2}, \\
\frac{\partial^{2} F_{\psi_{c}}}{\partial M \partial L}\left(M_{\psi_{c}}, L_{\psi_{c}}\right) & =0, \\
\frac{\partial^{2} F_{\psi_{c}}}{\partial L^{2}}\left(M_{\psi_{c}}, L_{\psi_{c}}\right) & =-\frac{16}{3} k_{1} a^{2} \sinh \frac{1}{2} \cosh \frac{1}{2}-8 k_{2} a \sinh \frac{1}{2},
\end{aligned}
$$

where $M_{\psi_{c}}=\max _{x \in \mathbb{S}^{1}}\left\{\psi_{c}(x)\right\}$ and $L_{\psi_{c}}=\min _{x \in \mathbb{S}^{1}}\left\{\psi_{c}(x)\right\}$. Moreover, $\left(M_{\psi_{c}}, L_{\psi_{c}}\right)$ is an isolated local maximum of $F_{\psi_{c}}$.

Proof. The expressions in (4.18) are obtained by a straightforward computation. The wave speed condition (1.19) is equivalent to the amplitude inequality $a>-\frac{3}{2} k_{2} / k_{1}$, which in turn implies that the Hessian matrix of $F_{\psi_{c}}$ at the critical point $\left(M_{\psi_{c}}, L_{\psi_{c}}\right)$ is diagonal and negative definite, which establishes its status as an isolated local maximum.

Lemma 4.5. [23] For any $v \in H^{1}\left(\mathbb{S}^{1}\right)$, we have

$$
\max _{x \in \mathbb{S}^{1}}|v(x)| \leq \sqrt{\frac{1}{2} \operatorname{coth} \frac{1}{2}}\|v\|_{H^{1}\left(\mathbb{S}^{1}\right)} .
$$

Moreover, $\sqrt{\frac{1}{2} \operatorname{coth} \frac{1}{2}}$ is the best constant, and equality holds if and only if $v=\psi_{c}(\cdot-\xi)$ for some $c>0$ and $\xi \in \mathbb{R}$, that is, if and only if $v$ has the profile of a peakon.

Lemma 4.6. [23] If $u \in C\left([0, T), H^{1}\left(\mathbb{S}^{1}\right)\right)$, then its spatial maximum and minimum values,

$$
M_{u(t)}=\max _{x \in \mathbb{S}^{1}}\{u(t, x)\}, \quad L_{u(t)}=\min _{x \in \mathbb{S}^{1}}\{u(t, x)\},
$$

are continuous functions of $t \in[0, T)$.

Lemma 4.7. Assume that $k_{1}>0, k_{2} \leq 0$, and the wave speed c satisfies (1.19). Then there exist a small neighborhood $\mathcal{U} \subset \mathbb{R}^{2}$ of $\left(M_{\psi_{c}}, L_{\psi_{c}}\right)$ and a $\delta>0$ such that whenever $u \in C\left([0, T), H^{s}\left(\mathbb{S}^{1}\right)\right), s>\frac{5}{2}$, is a periodic solution of the $\mathrm{gmCH}$ equation (2.1) with initial data $u_{0}=u(0, \cdot)$ satisfying $m_{0}=\left(1-\partial_{x}^{2}\right) u_{0}(x) \geq 0$ and $\left\|u_{0}-\psi_{c}\right\|_{H^{1}\left(\mathbb{S}^{1}\right)}<\delta$, then

$$
\left(M_{u(t)}, L_{u(t)}\right) \in \mathcal{U}, \quad \text { for all } \quad t \in[0, T) .
$$

Proof. According to Lemma 4.4, $\left(M_{\psi_{c}}, L_{\psi_{c}}\right)$ is an isolated local maximum of $F_{\psi_{c}}(M, L)$, and hence there exist neighborhoods $\mathcal{U} \subset \mathcal{V} \subset \mathbb{R}^{2}$ of $\left(M_{\psi_{c}}, L_{\psi_{c}}\right)$ and a constant $\alpha>0$ such that

$$
F_{\psi_{c}}(M, L) \leq-\alpha<0, \quad \text { for } \quad(M, L) \in \mathcal{V} \backslash \mathcal{U} .
$$

Lemma 4.2 implies continuity of the conserved functionals $H_{0}, H_{1}, H_{2}$ with respect to the $H^{1}\left(\mathbb{S}^{1}\right)$-norm. Thus, if $u_{0} \in H^{s}\left(\mathbb{S}^{1}\right), s>\frac{5}{2}$, is a small perturbation of $\psi_{c}$ in the $H^{1}\left(\mathbb{S}^{1}\right)$ norm such that $H_{j}\left[u_{0}\right]=H_{j}\left[\psi_{c}\right]+\epsilon_{j}$ for $j=0,1,2$, then the corresponding function (4.17), namely

$$
F_{u_{0}}(M, L)=F_{\psi_{c}}(M, L)+2 k_{2} L^{2} \epsilon_{0}+\left(k_{1}\left(\frac{4}{3} M^{2}-\frac{1}{3} L^{2}\right)+2 k_{2} M\right) \epsilon_{1}-\epsilon_{2}
$$


is a small perturbation of $F_{\psi_{c}}$. Thus, by choosing $\epsilon_{0}, \epsilon_{1}, \epsilon_{2}$ small enough, one ensures that

$$
\left\{(M, L) \in \mathcal{V} \mid F_{u_{0}}(M, L) \geq 0\right\} \subset \mathcal{U} .
$$

Hence, for $\delta>0$ sufficiently small, if $\left\|u_{0}-\psi_{c}\right\|_{H^{1}\left(\mathbb{S}^{1}\right)}<\delta$, then $\left(M_{\psi_{c}}, L_{\psi_{c}}\right) \in \mathcal{U}$. Let $u(t, x)$ be the corresponding periodic solution with initial data $u_{0}$. Lemmas 4.6 and 4.3 imply that $M_{u(t)}$ and $L_{u(t)}$ are continuous functions of $t \in[0, T)$ and that $F_{u(t)}\left(M_{u(t)}, L_{u(t)}\right) \geq 0$ for $t \in[0, T)$. This immediately implies that (4.20) holds, completing the proof.

Proof of Theorem 4.1. Let $u \in C\left([0, T), H^{s}\left(\mathbb{S}^{1}\right)\right), s \geq \frac{5}{2}$, be a periodic solution of the $\mathrm{gmCH}$ equation (2.1) with the initial data $m_{0}(x)=\left(1-\partial_{x}^{2}\right) u_{0}(x) \geq 0$. Given $\epsilon>0$, let $\mathcal{U}$ be a sufficiently small neighborhood of $\left(M_{\psi_{c}}, L_{\psi_{c}}\right)$ such that

$$
\left|M-M_{\psi_{c}}\right|<\frac{\epsilon^{2}}{8 a}, \quad \text { whenever } \quad(M, L) \in \mathcal{U} .
$$

Choose $\delta>0$ as in Lemma 4.7 so that (4.20) holds. Decreasing $\delta$ as necessary, we may also assume that

$$
\left|H_{1}[u]-H_{1}\left[\psi_{c}\right]\right|<\frac{1}{2} \epsilon^{2}, \quad \text { provided } \quad\left\|u(0, \cdot)-\psi_{c}\right\|_{H^{1}\left(S^{1}\right)}<\delta .
$$

Using Lemma 4.1, we conclude that, for $t \in[0, T)$,

$$
\begin{aligned}
\left\|u(t, \cdot)-\psi_{c}(\cdot-\xi(t))\right\|_{H^{1}\left(\mathbb{S}^{1}\right)}^{2} & =H_{1}[u]-H_{1}\left[\psi_{c}\right]-4 a\left(u(t, \xi(t))-M_{\psi_{c}}\right) \\
& \leq\left|H_{1}[u]-H_{1}\left[\psi_{c}\right]\right|+4 a\left|M_{u(t)}-M_{\psi_{c}}\right|<\epsilon^{2},
\end{aligned}
$$

where $\xi(t) \in \mathbb{R}$ is any point where $u(t, \xi(t))=M_{u(t)}$.

Finally, we state the corresponding results in the case $k_{1}>0$ and $k_{2}>0$, with positive and negative wave speeds, respectively. Theorems 4.2 and 4.3 are proved in a similar manner to the approach given in $[23,32]$, so we omit the details here.

Theorem 4.2. Assume that $k_{1}>0$ and $k_{2}>0$. Let $\psi_{c}$ be the peaked soliton defined in (4.1) with the traveling wave speed $c>0$. Then $\psi_{c}$ is orbitally stable in the following sense. Suppose that $u_{0} \in H^{s}\left(\mathbb{S}^{1}\right), s>\frac{5}{2}$, with $0 \not \equiv m_{0}(x)=\left(1-\partial_{x}^{2}\right) u_{0}(x) \geq 0$. Let $T>0$ be the maximal existence time of the corresponding periodic solution $u(t, x) \in C\left([0, T), H^{s}\left(\mathbb{S}^{1}\right)\right)$ to the initial data $u_{0}$. For every $\epsilon>0$, there is a $\delta>0$ such that if $\left\|u_{0}-\psi_{c}\right\|_{H^{1}\left(\mathbb{S}^{1}\right)}<\delta$, then

$$
\left\|u(t, \cdot)-\psi_{c}(\cdot-\xi(t))\right\|_{H^{1}\left(\mathbb{S}^{1}\right)}<\epsilon \quad \text { for } \quad t \in[0, T),
$$

where $\xi(t) \in \mathbb{R}$ is any point where the function $u(t, \cdot)$ attains its maximum.

Theorem 4.3. Assume that $k_{1}>0$ and $k_{2}>0$. Let $\psi_{c}$ be the peaked soliton defined in (4.1) with amplitude and traveling wave speed $c<0$ satisfying (1.16). Then $\psi_{c}$ is orbitally stable in the following sense. Suppose that $u_{0} \in H^{s}\left(\mathbb{S}^{1}\right), s>\frac{5}{2}$, with $0 \not \equiv m_{0}(x)=\left(1-\partial_{x}^{2}\right) u_{0}(x) \leq 0$. Let $T>0$ be the maximal existence time of the corresponding periodic solution $u(t, x) \in$ $C\left([0, T), H^{s}\left(\mathbb{S}^{1}\right)\right)$ to the initial data $u_{0}$. For every $\epsilon>0$, there is a $\delta>0$ such that if $\left\|u_{0}-\psi_{c}\right\|_{H^{1}\left(\mathbb{S}^{1}\right)}<\delta$, then

$$
\left\|u(t, \cdot)-\psi_{c}(\cdot-\xi(t))\right\|_{H^{1}\left(\mathbb{S}^{1}\right)}<\epsilon \quad \text { for } \quad t \in[0, T),
$$

where $\xi(t) \in \mathbb{R}$ is any point where the function $u(t, \cdot)$ attains its minimum.

\section{Appendix}

In this appendix, we provide the details of the proof of Theorem 2.1. 
Proof of Theorem 2.1. As always, we identify $\mathbb{S}^{1}$ with $[0,1)$ and consider $\psi_{c}(t, x)$ as a spatially periodic function on $\mathbb{R}$ with period one. Note first that $\psi_{c}$ is continuous on $\mathbb{S}^{1}$ with peak at $x=0$. Moreover, $\psi_{c}$ is smooth on $(0,1)$ and for $t>0$, its first order partial derivatives

$$
\partial_{x} \psi_{c}(t, x)=-a \sinh \zeta, \quad \partial_{t} \psi_{c}(t, x)=a c \sinh \zeta, \quad \text { where } \quad \zeta=\frac{1}{2}-(x-c t)+[x-c t],
$$

are both in $L^{\infty}\left(\mathbb{S}^{1}\right)$. Hence, if one denotes $\psi_{c, 0}(x)=\psi_{c}(0, x)$, then

$$
\lim _{t \rightarrow 0^{+}}\left\|\psi_{c}(t, \cdot)-\psi_{c, 0}(\cdot)\right\|_{W^{1, \infty}\left(\mathbb{S}^{1}\right)}=0 .
$$

Using (5.1), (5.2), and integration by parts, for any $\phi(t, x) \in C_{c}^{\infty}\left([0, \infty) \times \mathbb{S}^{1}\right)$, we have

$$
\begin{gathered}
\int_{0}^{\infty} \int_{\mathbb{S}^{1}}\left(\psi_{c} \partial_{t} \phi+\frac{1}{3} k_{1} \psi_{c}^{3} \partial_{x} \phi+\frac{1}{3} k_{1}\left(\partial_{x} \psi_{c}\right)^{3} \phi+\frac{1}{2} k_{2} \psi_{c}^{2} \partial_{x} \phi\right) d x d t+\int_{\mathbb{S}^{1}} \psi_{c, 0}(x) \phi(0, x) d x \\
\quad=-\int_{0}^{\infty} \int_{\mathbb{S}^{1}} \phi\left(\partial_{t} \psi_{c}+k_{1} \psi_{c}^{2} \partial_{x} \psi_{c}-\frac{1}{3} k_{1}\left(\partial_{x} \psi_{c}\right)^{3}+k_{2} \psi_{c} \partial_{x} \psi_{c}\right) d x d t \\
\quad=\int_{0}^{\infty} \int_{\mathbb{S}^{1}} \phi\left(\left(k_{1} a^{3}-a c\right) \sinh \zeta+\frac{2}{3} k_{1} a^{3} \sinh ^{3} \zeta+k_{2} a^{2} \sinh \zeta \cosh \zeta\right) d x d t
\end{gathered}
$$

In addition, the explicit form (2.4) of the Green's function $G(x)$ for the periodic case, along with (5.1) and the proof of Theorem 4.1 in [32], implies that

$$
\begin{gathered}
\int_{0}^{\infty} \int_{\mathbb{S} 1}\left[k_{1} G(x) *\left(\frac{2}{3} \psi_{c}^{3}+\psi_{c}\left(\partial_{x} \psi_{c}\right)^{2}\right) \partial_{x} \phi-\frac{1}{3} k_{1} G(x) *\left(\partial_{x} \psi_{c}\right)^{3} \phi\right] d x d t \\
=k_{1} a^{3} \int_{0}^{\infty} \int_{\mathbb{S}^{1}} \phi G(x) *\left(2 \sinh \zeta+\frac{7}{3} \sinh ^{3} \zeta\right) d x d t \\
\quad-k_{1} a^{3} \int_{0}^{\infty} \int_{\mathbb{S}^{1}} \phi G_{x}(x) *\left(\cosh \zeta \sinh ^{2} \zeta\right) d x d t \\
=\frac{2}{3} k_{1} a^{3} \int_{0}^{\infty} \int_{\mathbb{S}^{1}} \phi\left(\sinh ^{2} \frac{1}{2} \sinh \zeta-\sinh ^{3} \zeta\right) d x d t .
\end{gathered}
$$

Now, we compute directly

$$
\begin{aligned}
\int_{0}^{\infty} \int_{\mathbb{S}^{1}} k_{2} G *\left(\psi_{c}^{2}+\frac{1}{2}\left(\partial_{x} \psi_{c}\right)^{2}\right) \partial_{x} \phi d x d t & =\int_{0}^{\infty} \int_{\mathbb{S}^{1}} k_{2} \phi G * \partial_{x}\left(\psi_{c}^{2}+\frac{1}{2}\left(\partial_{x} \psi_{c}\right)^{2}\right) d x d t \\
& =-\frac{3}{2} k_{2} a^{2} \int_{0}^{\infty} \int_{\mathbb{S}^{1}} \phi G * \sinh (2 \zeta) d x d t .
\end{aligned}
$$

Thus, when $x>c t$,

$$
\begin{array}{rl}
G & * \sinh (2 \zeta)(t, x) \\
& =\frac{1}{2 \sinh \frac{1}{2}} \int_{\mathbb{S}^{1}} \cosh \left(\frac{1}{2}-(x-z)+[x-z]\right) \cdot \sinh (1-2(z-c t)+2[z-c t]) d z \\
& =\frac{2}{3}\left[\cosh \left(\frac{1}{2}\right) \sinh \left(\frac{1}{2}-(x-c t)\right)-\sinh \left(\frac{1}{2}-(x-c t)\right) \cosh \left(\frac{1}{2}-(x-c t)\right)\right] .
\end{array}
$$

In a similar manner, for $x<c t$, we have

$$
\begin{aligned}
& G * \sinh (2 \zeta)(t, x) \\
& \quad=\frac{2}{3}\left[-\cosh \left(\frac{1}{2}\right) \sinh \left(\frac{1}{2}+(x-c t)\right)+\sinh \left(\frac{1}{2}+(x-c t)\right) \cosh \left(\frac{1}{2}+(x-c t)\right)\right] .
\end{aligned}
$$

Plugging (5.6) and (5.7) into (5.5) yields

$$
\begin{aligned}
\int_{0}^{\infty} \int_{\mathbb{S}^{1}} k_{2} G *\left(\psi_{c}^{2}\right. & \left.+\frac{1}{2}\left(\partial_{x} \psi_{c}\right)^{2}\right) \partial_{x} \phi d x d t \\
& =k_{2} a^{2} \int_{0}^{\infty} \int_{\mathbb{S}^{1}} \phi \sinh \zeta\left(\cosh \zeta-\cosh \frac{1}{2}\right) d x d t
\end{aligned}
$$


In view of (5.3), (5.4), and (5.8), we have

$$
\begin{gathered}
\int_{0}^{\infty} \int_{\mathbb{S}^{1}}\left[\psi_{c} \partial_{t} \phi+\frac{1}{3} k_{1} \psi_{c}^{3} \partial_{x} \phi+\frac{1}{3} k_{1}\left(\partial_{x} \psi_{c}\right)^{3} \phi+\frac{1}{2} k_{2} \psi_{c}^{2} \partial_{x} \phi\right. \\
+k_{1} G(x) *\left(\frac{2}{3} \psi_{c}^{3}+\psi_{c}\left(\partial_{x} \psi_{c}\right)^{2}\right) \partial_{x} \phi-\frac{1}{3} k_{1} G(x) *\left(\partial_{x} \psi_{c}\right)^{3} \phi \\
\left.+k_{2} G *\left(\psi_{c}^{2}+\frac{1}{2}\left(\partial_{x} \psi_{c}\right)^{2}\right) \partial_{x} \phi\right] d x d t+\int_{\mathbb{S}^{1}} \psi_{c, 0}(x) \phi(0, x) d x \\
=\int_{0}^{\infty} \int_{\mathbb{S}^{1}} \phi\left[\left(k_{1} a^{3}-a c\right) \sinh \zeta+\frac{2}{3} k_{1} a^{3} \sinh ^{2} \frac{1}{2} \cdot \sinh \zeta+k_{2} a^{2} \cosh \frac{1}{2} \sinh \zeta\right] d x d t \\
=\int_{0}^{\infty} \int_{\mathbb{S}^{1}} \phi a\left[\frac{1}{3} k_{1}\left(1+2 \cosh ^{2} \frac{1}{2}\right) a^{2}+k_{2} a \cosh \frac{1}{2}-c\right] \sinh \zeta d x d t .
\end{gathered}
$$

If $a$ takes the value given in (1.16), then

$$
\frac{1}{3} k_{1}\left(1+2 \cosh ^{2} \frac{1}{2}\right) a^{2}+k_{2} a \cosh \frac{1}{2}-c=0,
$$

which implies that

$$
\begin{aligned}
\int_{0}^{\infty} \int_{\mathbb{S}^{1}}\left[\psi_{c} \partial_{t} \phi+\right. & \frac{1}{3} k_{1} \psi_{c}^{3} \partial_{x} \phi+\frac{1}{3} k_{1}\left(\partial_{x} \psi_{c}\right)^{3} \phi+\frac{1}{2} k_{2} \psi_{c}^{2} \partial_{x} \phi \\
+ & k_{1} G(x) *\left(\frac{2}{3} \psi_{c}^{3}+\psi_{c}\left(\partial_{x} \psi_{c}\right)^{2}\right) \partial_{x} \phi-\frac{1}{3} k_{1} G(x) *\left(\partial_{x} \psi_{c}\right)^{3} \phi \\
+ & \left.k_{2} G(x) *\left(\psi_{c}^{2}+\frac{1}{2}\left(\partial_{x} \psi_{c}\right)^{2}\right) \partial_{x} \phi\right] d x d t+\int_{\mathbb{S}^{1}} \psi_{c, 0}(x) \phi(0, x) d x=0
\end{aligned}
$$

for any $\phi(t, x) \in C_{c}^{\infty}\left([0, \infty) \times \mathbb{S}^{1}\right)$. This completes the proof.

Acknowledgements. The authors thank the referee for valuable comments and suggestions. The work of X.C. Liu is supported by NSF-China grant 11001220. The work of Y. Liu is partially supported by NSF grant DMS-1207840. The work of P.J. Olver is partially supported by NSF grant DMS-1108894. The work of C.Z. Qu is supported in part by the NSF-China for Distinguished Young Scholars grant-10925104 and Ph.D. Programs Foundation of Ministry of Education of China-20106101110008.

\section{REFERENCES}

[1] R. Camassa and D.D. Holm, An integrable shallow water equation with peaked solitons, Phys. Rev. Lett., 71 (1993), 1661-1664.

[2] K.S. Chou AND C.Z. Qu, Integrable equations arising from motions of plane curves I, Physica D, 162 (2002), 9-33.

[3] A. Constantin, Existence of permanent and breaking waves for a shallow water equation: a geometric approach, Ann. Inst. Fourier (Grenoble), 50 (2000), 321-362.

[4] A. Constantin, The trajectories of particles in Stokes waves, Invent. Math., 166 (2006), 523-535.

[5] A. Constantin And J. Escher, Wave breaking for nonlinear nonlocal shallow water equations, Acta Math., 181 (1998), 229-243.

[6] A. Constantin and J. Escher, Global existence and blow-up for a shallow water equation, Ann. Scuola Norm. Sup. Pisa, 26 (1998), 303-328.

[7] A. Constantin and J. Escher, On the blow-up rate and the blow-up set of breaking waves for a shallow water equation, Math. Z., 233 (2000), 75-91.

[8] A. Constantin And J. Escher, Analyticity of periodic traveling free surface water waves with vorticity, Ann. Math., 173 (2011), 559-568. 
[9] A. Constantin and D. Lannes, The hydrodynamical relevance of the Camassa-Holm and Degasperis-Procesi equations, Arch. Rational Mech. Anal., 192 (2009), 165-186.

[10] A. Constantin And L. Molinet, Orbital stability of solitary waves for a shallow water equation, Physica D, 157 (2001), 75-89.

[11] A. Constantin and W. Strauss, Stability of peakons, Commun. Pure Appl. Math., 53 (2000), 603-610.

[12] A. Degasperis, D.D. Holm, and A.N.W. Hone, Integrable and non-integrable equations with peakons, Nonlinear physics: theory and experiment, II (Gallipoli, 2002), 37 (World Sci. Publ., River Edge, NJ, 2003).

[13] K. El Dika And L. Molinet, Stability of multipeakons, Ann. Inst. Henri Poincaré Anal. Non Lin'eaire, 18 (2009), 1517-1532.

[14] M. Faquir, M.A. Manna, And A. Neveu, An integrable equation governing short waves in a long-wave model, Proc. R. Soc. A, 463 (2007), 1939-1954.

[15] A.S. FokAs, The Korteweg-de Vries equation and beyond, Acta Appl. Math., 39 (1995), 295-305.

[16] A.S. FokAs, On a class of physically important integrable equations, Physica D, $\mathbf{8 7}$ (1995), 145-150.

[17] B. Fuchssteiner, Some tricks from the symmetry-toolbox for nonlinear equations: generalizations of the Camassa-Holm equation, Physica D, 95 (1996), 229-243.

[18] B. Fuchssteiner And A.S. Fokas, Symplectic structures, their Bäcklund transformations and hereditary symmetries, Physica D, 4 (1981/1982), 47-66.

[19] G.L. Gui, Y. Liu, P.J. Olver, and C.Z. Qu, Wave-breaking and peakons for a modified Camassa-Holm equation, Commun. Math. Phys., 319 (2013), 731-759.

[20] R.E. Goldstein and D.M. Petrich, The Korteweg-de Vries hierarchy as dynamics of closed curves in the plane, Phys. Rev. Lett., 67 (1991), 3203-3206.

[21] H. Holden and X. RAYnaud, A convergent numerical scheme for the Camassa-Holm equation based on multipeakons, Discrete Cont. Dyn. Syst. A, 14 (2006), 505-523.

[22] R.S. Johnson, Camassa-Holm, Korteweg-de Vries and related models for water waves, J. Fluid Mech., 455 (2002), 63-82.

[23] J. Lenells, Stability of periodic peakons, Int. Math. Res. Not., 10 (2004), 485-499.

[24] Y.A. Li AND P.J. Olver, Well-posedness and blow-up solutions for an integrable nonlinearly dispersive model wave equation, J. Diff. Eq., 162 (2000), 27-63.

[25] Y.A. Li, P.J. Olver, AND P. Rosenau, Non-analytic solutions of nonlinear wave models, in: Nonlinear Theory of Generalized Functions, M. Grosser, G. Höormann, M. Kunzinger, and M. Oberguggenberger, eds., Research Notes in Mathematics, vol. 401, Chapman and Hall/CRC, New York, 1999, pp. 129-145.

[26] Z.W. Lin AND Y. Liu, Stability of peakons for the Degasperis-Procesi equation, Commun. Pure Appl. Math., 62 (2009), 125-146.

[27] X.C. LiU, Y. LiU, AND C.Z. QU, Orbital stability of the train of peakons for an integrable modified Camassa-Holm equation, Adv. Math., 255 (2014), 1-37.

[28] P.J. Olver, Applications of Lie Groups to Differential Equations, Second Edition, Graduate Texts in Mathematics, vol. 107, Springer-Verlag, New York, 1993.

[29] P.J. Olver and P. Rosenau, Tri-Hamiltonian duality between solitons and solitarywave solutions having compact support, Phys. Rev. E, 53 (1996), 1900-1906.

[30] Z. QIAO, A new integrable equation with cuspons and W/M-shape-peaks solitons, $J$. Math. Phys., 47 (2006), 112701, 9pp.

[31] Z. QIaO, B. XiA, AND J.B. Li, A generalized Camassa-Holm equation and its peakon solutions, http://xxx. lanl. gov/abs/1205. 2028.

[32] C.Z. QU, X.C. LIU, AND Y. LiU, Stability of peakons for an integrable modified Camassa-Holm equation, Commun. Math. Phys., 322 (2013), 967-997.

[33] C.Z. Qu, Y. Zhang, X.C. LiU, AND Y. LiU, Orbital stability of periodic peakons to a generalized $\mu$-Camassa-Holm equation, Arch. Rational Mech. Anal., 211 (2014), 593-617.

[34] T. Schäfer and C.E. Wayne, Propagation of ultra-short optical pulses in cubic nonlinear media, Physica D, 196 (2004), 90-105. 
[35] J. F. Toland, Stokes waves, Topol. Methods Nonlinear Anal., 7 (1996), 1-48.

XiaOchuan LiU

Department of Mathematics, Northwest University, Xi'An 710069, P. R. China

E-mail address: liuxc@nwu.edu.cn

Yue LiU

Department of Mathematics, University of Texas, Arlington, TX 76019, USA

E-mail address: yliu@uta.edu

Peter J. Olver

School of Mathematics, University of Minnesota, Minneapolis, MN 55455, USA

E-mail address: olver@umn.edu

Changzheng QU

Department of Mathematics, Ningbo University, Ningbo 315211, P. R. China

E-mail address: quchangzheng@nbu.edu.cn 